\title{
An Expedient Formal Total Synthesis of (-)-Diazonamide A via a Powerful, Stereoselective $O$-Aryl to $C$-Aryl Migration to form the C10 Quaternary Center.
}

Chi-Ming Cheung, Frederick W. Goldberg, Philip Magnus* Claire J. Russell and Rachel Turnbull and Vince Lynch

Department of Chemistry and Biochemistry, University of Texas at Austin, 1 University Station A5300, Austin, Texas 78712-1167,USA; E-mail: p.magnus@mail.utexas.edu

\section{Experimental}

Melting points (m.p.) were measured using a Thomas-Hoover capillary tube melting point apparatus and are uncorrected. Infrared spectra were recorded on a Nicolet Fourier Transform Spectrometer (225 to $4400 \mathrm{~cm}^{-1}$ ). The samples were prepared as evaporated films on sodium chloride disks unless stated otherwise. ${ }^{1} \mathrm{H}$ and ${ }^{13} \mathrm{C}$ NMR spectra were recorded on a General Electric QE-300 Spectrometer operating at ambient probe temperature using an internal deuterium lock (300 MHz for ${ }^{1} \mathrm{H}$ NMR and $75 \mathrm{MHz}$ for ${ }^{13} \mathrm{C} \mathrm{NMR}$ ), unless stated otherwise. Chemical shifts are reported in parts per million (d) at lower frequencies relative to tetramethylsilane (TMS) and are referenced internally. Mass spectra including chemical ionisation (C.I.) and HRMS were recorded on a VG ZAB2E or a Finnigan TSQ70 quadrupole mass spectrometer. Accurate mass measurements are correct to \pm 0.001 . Flash chromatography was performed using EMD silica gel (40-63 microns). Analytical thin layer chromatography (TLC) was carried out using Merck ${ }^{\circledR}$ silica gel 60 F254 plates with visualisation using either UV light, vanillin or potassium permanganate.

The solvents used were either distilled over appropriate drying agents, or of analytical grade. All other commercially available reagents were purified as necessary following standard procedures.

\section{General Procedures}

Method A - Solid Phase rearrangement of Cbz-protected macrolactam ethers 31 and 32; and Boc-protected macrocyclic etherlactams $\mathbf{4 1}$ and $\mathbf{4 2 .}$ 
Ethers 31 and 32, or 41 and 42 (0.01 mmol, 1 eq., as a mixture or single diastereomer, as indicated in Table 1) were kept at the time and temperature indicated in Table 1 before it was analyzed using HPLC.

Method B - Thermal rearrangement of Cbz-protected macrolactam ethers 31 and 32; and Bocprotected macrocyclic etherlactams $\mathbf{4 1}$ and $\mathbf{4 2}$.

Ethers 31 and 32, or $\mathbf{4 1}$ and $\mathbf{4 2}$ (0.01 mmol, 1 eq., as a mixture or single diastereomer, as indicated in Table 1) was dissolved in the solvent $(1 \mathrm{ml})$ and stirred at the temperature and time described in Table 1. The solvent was then evaporated and the residue analyzed using HPLC.

Method C - Acid catalyzed rearrangement of Cbz-protected macrolactam ethers 31 and 32; and Boc-protected macrocyclic etherlactams $\mathbf{4 1}$ and $\mathbf{4 2}$

Ethers 31 and 32, or 41 and 42 (0.01 mmol, 1 eq., as a mixture or single diastereomer, as indicated in Table 1) was dissolved in the solvent (1 ml) and a catalytic amount of acid (3 mol\%) added. The mixture was then stirred at the temperature and time described in Table 1, before the solvent was evaporated and the residue was portioned between ethyl acetate $(2 \mathrm{ml})$ and saturated aqueous $\mathrm{NaHCO}_{3}(2 \mathrm{ml})$. The aqueous phase was extracted with ethyl acetate $(2 \times 2 \mathrm{ml})$. The organic phases were combined, dried $\left(\mathrm{Na}_{2} \mathrm{SO}_{4}\right)$, filtered and evaporated in vacuo. Analysis was carried out using HPLC.

\section{Di-peptide of (S) $N$-t-Butoxycarbonyl Valine Serine methyl ester $18 .^{1}$}

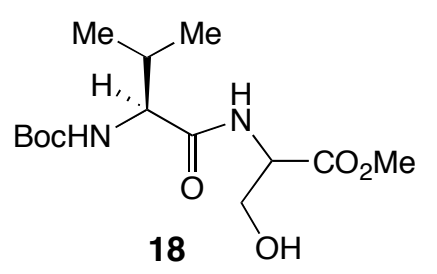

To a mechanically stirred solution of Boc- $S$-valine (63 g, $0.29 \mathrm{~mol}, 1.0$ equiv) in THF $(1.2 \mathrm{~L})$ at $-30{ }^{\circ} \mathrm{C}$, was added triethylamine $(85 \mathrm{~mL}, 62 \mathrm{~g}, 0.61 \mathrm{~mol}, 2.1$ equiv) and isobutyl chloroformate (42 mL, $44 \mathrm{~g}, 0.32 \mathrm{~mol}, 1.1$ equiv) and the mixture stirred for for $1 \mathrm{~h}$ (thick white mixture). ( \pm )-Serine methyl ester hydrochloride (50 g, 0.32 mol, 1.1 equiv) was added and the mixture was stirred at $-30{ }^{\circ} \mathrm{C}$ for $3 \mathrm{~h}$ and warmed to ambient temperature overnight. The THF was evaporated and the residue was portioned between ethyl acetate $(2 \mathrm{~L})$ and water $(1 \mathrm{~L})$. The 
aqueous phase was extracted with ethyl acetate $(2 \times 1 \mathrm{~L})$. The organic phases were combined, dried $\left(\mathrm{Na}_{2} \mathrm{SO}_{4}\right)$, filtered and evaporated in vacuo to give $\mathbf{1 8}$ (colorless oil) as a mixture of two diastereomers (93 g, 99\%). ${ }^{1} \mathrm{H} \mathrm{NMR}\left(\mathrm{CDCl}_{3}, 300 \mathrm{MHz}\right) \delta$ 0.85-1.03 (6H, m), $1.40(9 \mathrm{H}, \mathrm{s}), 2.20$ $(1 \mathrm{H}, \mathrm{m}), 3.76(2 \mathrm{H}, \mathrm{s}), 3.90(3 \mathrm{H}, \mathrm{m}), 4.64(1 \mathrm{H}, \mathrm{m}), 5.15(1 \mathrm{H}, \mathrm{m}), 7.95(1 \mathrm{H}, \mathrm{m})$.

\section{Formation of 19.}

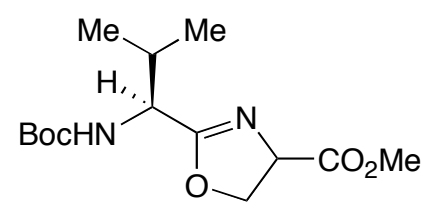

19 (68\%)

A solution of 18 (31 g, $97 \mathrm{mmol}, 1.0$ equiv) in THF (500 mL) was added to a preformed solution of the Burgess reagent ${ }^{2}$ (38 g, 159 mmol, 1.6 equiv) in THF (500 mL) and the reaction mixture was heated to reflux for $12 \mathrm{~h}$. After cooling to ambient temperature, the solvent was evaporated in vacuo and the residue was purified by column chromatography ( $20 \%$ to $50 \%$ ethyl acetate in hexanes) to give 19 (colorless oil) as a mixture of two diastereomers $(19.7 \mathrm{~g}, 68 \%) .{ }^{1} \mathrm{H}$ NMR $\left(\mathrm{CDCl}_{3}\right) \delta$ 0.80-1.00 (6H, m), $1.40(9 \mathrm{H}, \mathrm{s}), 2.14(1 \mathrm{H}, \mathrm{m}), 3.80(3 \mathrm{H}, \mathrm{s}), 4.32(1 \mathrm{H}, \mathrm{m}), 4.40-$ $4.60(2 \mathrm{H}, \mathrm{m}), 4.78(1 \mathrm{H}, \mathrm{m}), 5.10(1 \mathrm{H}, \mathrm{m})$.

\section{Formation of oxazole methyl ester $20 .^{3}$}

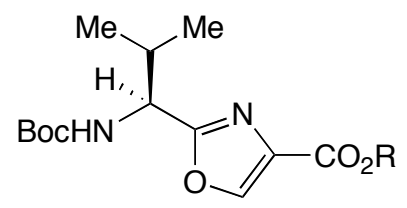

$20(\mathrm{R}=\mathrm{Me}, 79 \%)$

$21(R=H, 100 \%)$

To a solution of 19 (19.7 g, $65.6 \mathrm{mmol}, 1.0$ equiv) in dichloromethane (400 mL) at $0{ }^{\circ} \mathrm{C}$, was added DBU (11.8 mL, $12.0 \mathrm{~g}, 78.7 \mathrm{mmol}, 1.2$ equiv) followed by dropwise addition of bromotrichloromethane ( $8.4 \mathrm{~mL}, 85.3 \mathrm{mmol}, 1.3 \mathrm{equiv})$ over $20 \mathrm{~min}$. The mixture was warmed to ambient temperature and was stirred for an additional $14 \mathrm{~h}$. The reaction mixture was washed with sat. aq. $\mathrm{NH}_{4} \mathrm{Cl}(150 \mathrm{~mL})$, and the aqueous phase was extracted with dichloromethane $(2 \mathrm{x}$ $100 \mathrm{~mL})$. The combined organic phases were dried $\left(\mathrm{Na}_{2} \mathrm{SO}_{4}\right)$, filtered and concentrated in vacuo. The residue was purified by column chromatography (35\% ethyl acetate in hexanes) and gave 20 
as a white solid $(15.4 \mathrm{~g}, 79 \%) .{ }^{1} \mathrm{H} \mathrm{NMR}\left(\mathrm{CDCl}_{3}\right) \delta 0.90(6 \mathrm{H}, \mathrm{m}), 1.40(9 \mathrm{H}, \mathrm{s}), 2.20(1 \mathrm{H}, \mathrm{m})$, $3.92(3 \mathrm{H}, \mathrm{s}), 4.78(1 \mathrm{H}, \mathrm{m}), 5.24(1 \mathrm{H}, \mathrm{m}), 8.20(1 \mathrm{H}, \mathrm{s})$.

\section{Saponification of 20 to give 21.}

To a solution of $\mathbf{2 0}$ (15.4 g, $51.6 \mathrm{mmol}, 1.0$ equiv) in THF (350 mL) was added (1 M) $\mathrm{NaOH}$ (129 mL, $129 \mathrm{mmol}, 2.5$ equiv). The reaction mixture was stirred for $14 \mathrm{~h}$, then the solvent was evaporated in vacuo and the residue was diluted with ethyl acetate $(200 \mathrm{~mL})$ and (1 M) HCL (150 mL). The aqueous phase was extracted with ethyl acetate $(2 \times 100 \mathrm{~mL})$ and the combined organic phases were dried $\left(\mathrm{Na}_{2} \mathrm{SO}_{4}\right)$, filtered and concentrated in vacuo. The residue was azeotroped with toluene $(2 \times 35 \mathrm{~mL})$ to give 21 as a white solid $(14.7 \mathrm{~g}, 100 \%)$. ${ }^{1} \mathrm{H}$ NMR $\left(\mathrm{CDCl}_{3}\right) \delta$ 0.88-0.92 (6H, m), $1.40(9 \mathrm{H}, \mathrm{s}), 2.10-2.22(1 \mathrm{H}, \mathrm{m}), 4.82(1 \mathrm{H}, \mathrm{m}), 6.20(1 \mathrm{H}, \mathrm{m}), 8.30$ $(1 \mathrm{H}, \mathrm{s}), 11.5(1 \mathrm{H}, \mathrm{s}, \mathrm{br})$.

\section{Synthesis of 7-bromoisatin $23^{4}$ and $N$-methoxymethyl-7-bromoisatin $24 .^{5}$}

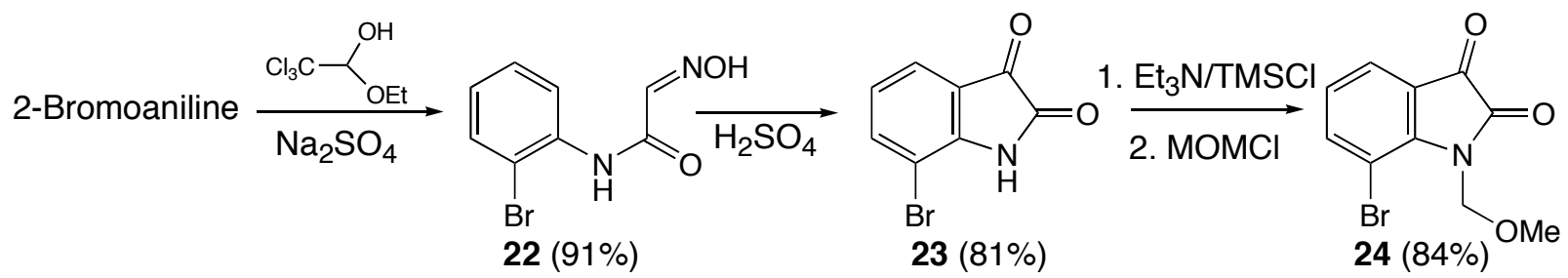

To a well stirred solution of 2,2,2 trichloro-1-ethoxyethanol (50 g, $0.26 \mathrm{~mol}, 1.5$ equiv), sodium sulfate (163 g, $1.15 \mathrm{~mol}, 6.67$ equiv) and hydroxylamine hydrochloride (40 g, $0.57 \mathrm{~mol}$, 3.33 equiv) in water $(900 \mathrm{~mL})$ was added a mixture of 2-bromoaniline (29.6 g, $0.17 \mathrm{mmol}, 1.0$ equiv) and $36 \% \mathrm{HCl}(25 \mathrm{~mL})$ in water $(100 \mathrm{~mL})$ with vigorous stirring. The reaction was gently heated to $37{ }^{\circ} \mathrm{C}$ during $2.5 \mathrm{~h}$, at which time a yellow solid appeared. The reaction mixture was then heated to $45^{\circ} \mathrm{C}$ over $2 \mathrm{~h}$, and was cooled to ambient temperature. The precipitate formed was collected by filtration, washed with water $(200 \mathrm{~mL})$, and dried under vacuum to yield crude $22(38 \mathrm{~g}, 91 \%)$.

Crude 22 (40 g, $0.16 \mathrm{~mol}$ ) was added portion-wise to a rapidly stirred solution of concentrated sulfuric acid $\left(180 \mathrm{~mL}\right.$ ) warmed to $55^{\circ} \mathrm{C}$ (at a rate to keep the reaction temperature between $55-70{ }^{\circ} \mathrm{C}$. The resulting solution was warmed to $70{ }^{\circ} \mathrm{C}$ and stirred for $20 \mathrm{~min}$ and then cooled to ambient temperature. The purplish-red mixture was poured carefully onto crushed ice 
(ca. $900 \mathrm{~g}$ ) and the mixture was allowed to stand for $1 \mathrm{~h}$. The orange precipitate was collected by filtration, washed with water $(200 \mathrm{~mL})$, and then dried under vacuum and gave $23(30 \mathrm{~g}, 81 \%)$. ${ }^{1} \mathrm{H} \mathrm{NMR}\left(\mathrm{CDCl}_{3}\right) \delta 7.06(1 \mathrm{H}, \mathrm{t}, J=7.5 \mathrm{~Hz}), 7.58(1 \mathrm{H}, \mathrm{d}, J=7.5 \mathrm{~Hz}), 7.73(1 \mathrm{H}, \mathrm{d}, J=8.0 \mathrm{~Hz})$, $7.98(1 \mathrm{H}, \mathrm{s}, \mathrm{br})$.

Triethylamine (46.3 mL, $33.6 \mathrm{~g}, 0.33 \mathrm{~mol}, 5.0$ equiv) was added to a solution of 23 (15 g, $0.06 \mathrm{~mol}, 1.0$ equiv) in THF (250 mL) (insoluble) followed by chlorotrimethylsilane $(42 \mathrm{~mL}$, $36.0 \mathrm{~g}, 0.33 \mathrm{~mol}, 5.0$ equiv). The reaction mixture fumed and went bright orange and a precipitate was formed. The reaction mixture was heated at reflux for $2 \mathrm{~h}$ (reaction was covered by foil). The reaction was cooled to $0^{\circ} \mathrm{C}$ and a solution of $\mathrm{MOMCl}(29.6 \mathrm{~mL}, 31.4 \mathrm{~g}, 0.33 \mathrm{~mol}$, 5.0 equiv) in THF $(30 \mathrm{~mL})$ added at $0{ }^{\circ} \mathrm{C}$. The mixture was stirred at $0{ }^{\circ} \mathrm{C}$ for $1 \mathrm{~h}$ and then heated at reflux for $2 \mathrm{~h}$. The reaction mixture was concentrated under in vacuo and the residue taken up in ethyl acetate $(400 \mathrm{~mL})$, washed with water $(250 \mathrm{~mL})$, and extracted with ethyl acetate $(3 \times 250$ $\mathrm{mL})$. The combined ectracts were washed with brine $(500 \mathrm{~mL})$, dried $\left(\mathrm{Na}_{2} \mathrm{SO}_{4}\right)$, filtered and concentrated. The solid was pre-absorbed onto silica. Purification by column chromatography over silica gel eluting with 30:40:30\% ethyl acetate:hexanes:dichloromethane gave $\mathbf{2 4}$ as an orange solid (15.0 g, 84\%). ${ }^{1} \mathrm{H}$ NMR $\left(\mathrm{CDCl}_{3}\right) \delta 3.42(3 \mathrm{H}, \mathrm{s}), 5.52(2 \mathrm{H}, \mathrm{s}), 7.06(1 \mathrm{H}, \mathrm{t}, J=8.0$ $\mathrm{Hz}), 7.66(1 \mathrm{H}, \mathrm{dd}, J=7.5,1.5 \mathrm{~Hz}), 7.78(1 \mathrm{H}, \mathrm{dd}, J=8.0,1.5 \mathrm{~Hz}){ }^{5}$

\section{5-(7-Bromo-3-hydroxy-1-methoxymethyl-2-oxo-2,3-dihydro-1H-indol-3-yl)-2-[1-(2,2-}

dimethyl-propionyloxy)-2-methyl-propyl]-oxazole-4-carboxylic acid methyl ester 25 and 26.

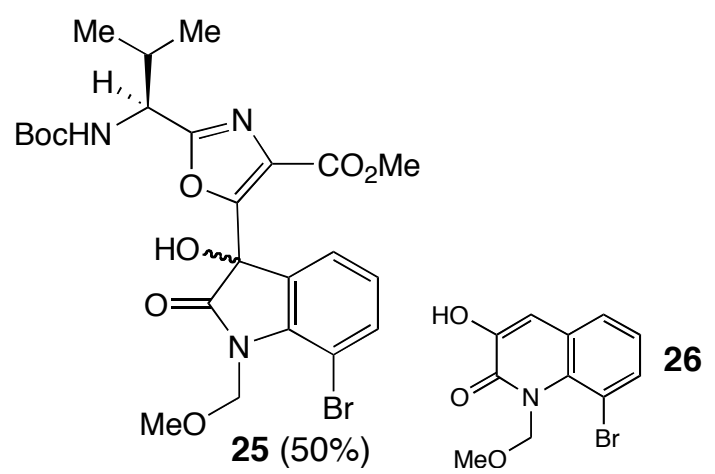

$t$-Butyl lithium (31.0 $\mathrm{mL}, 1.7 \mathrm{M}$ in hexanes, $52.7 \mathrm{mmol}$ ) was added dropwise to a solution of 21 (4.41 g, $15.5 \mathrm{mmol}$, dried by repeated in vacuo concentrations from toluene) in THF (100 mL) and HMPA (9.27 mL, $52.7 \mathrm{mmol}$, previously distilled from sodium and stored over sieves) at $-78{ }^{\circ} \mathrm{C}$ under argon and the reaction mixture was stirred for $1 \mathrm{~h}$. A solution of 24 
(6.62 g, $24.5 \mathrm{mmol}$, dried by repeated in vacuo concentrations from toluene) in THF (40 mL) was added over 40 minutes via syringe pump and then additional THF $(10 \mathrm{~mL})$ was used to rinse the syringe into the reaction mixture. The reaction mixture was stirred for one hour at $-78{ }^{\circ} \mathrm{C}$, and was quenched while still cold with sat. aq. $\mathrm{NH}_{4} \mathrm{Cl}$, allowed to warm to room temperature, taken up in ethyl acetate and water and extracted with ethyl acetate. The aqueous layer was carefully acidified with $2 \mathrm{M}$ aq. $\mathrm{HCl}$ and extracted four times with ethyl acetate. The combined organic layers were washed three times with sat. aq. $\mathrm{NH}_{4} \mathrm{Cl}$ and with sat. brine, the combined washes were back extracted with ethyl acetate and the combined extracts were dried $\left(\mathrm{Na}_{2} \mathrm{SO}_{4}\right)$, filtered and concentrated in vacuo to obtain the crude hydroxy acid intermediate. $\mathrm{TMSCHN}_{2}(15$ $\mathrm{mL}, 2 \mathrm{M}$ in $\mathrm{Et}_{2} \mathrm{O}, 20 \mathrm{mmol}$ ) was added to a solution of the hydroxy acid intermediate in methanol $(50 \mathrm{~mL})$ and toluene $(50 \mathrm{~mL})$ at $0{ }^{\circ} \mathrm{C}$, the reaction mixture was stirred at room temperature for 10 minutes, concentrated in vacuo and purified by flash column, eluting with 1:2 ethyl acetate:hexane to obtain $\mathbf{2 5}(4.37 \mathrm{~g}, 50 \%)$ as a mixture of diastereoisomers. Pale yellow solid, m.p. $62-66{ }^{\circ} \mathrm{C}$. IR 3325, 2967, 1746, 1716, 1600, 1580, $1507 \mathrm{~cm}^{-1} .{ }^{1} \mathrm{H}\left(300 \mathrm{MHz}, \mathrm{CDCl}_{3}\right)$ $\delta 7.72(0.5 \mathrm{H}, \mathrm{s}), 7.66(0.5 \mathrm{H}, \mathrm{s}), 7.54(1 \mathrm{H}, \mathrm{d}, J=7.9), 7.29-7.23(1 \mathrm{H}, \mathrm{m}), 6.99(0.5 \mathrm{H}, \mathrm{t}, J=7.7)$, $6.98(0.5 \mathrm{H}, \mathrm{t}, J=7.7), 5.51-5.44(2 \mathrm{H}, \mathrm{m}), 5.15-5.07(1 \mathrm{H}, \mathrm{m}), 4.66-4.55(1 \mathrm{H}, \mathrm{m}), 4.04(3 \mathrm{H}, \mathrm{s})$, $3.39(1.5 \mathrm{H}, \mathrm{s}), 3.38(1.5 \mathrm{H}, \mathrm{s}), 2.03-1.95(1 \mathrm{H}, \mathrm{m}), 1.39(4.5 \mathrm{H}, \mathrm{s}), 1.37(4.5 \mathrm{H}, \mathrm{s}), 0.83-0.77(6 \mathrm{H}$, m). ${ }^{13} \mathrm{C}\left(75 \mathrm{MHz}, \mathrm{CDCl}_{3}\right) \delta 174.5,174.4,164.4,164.3,163.1,163.1,154.9,139.6,139.4,136.5$, 130.9, 130.7, 130.1, 129.9, 125.1, 125.0, 124.0, 123.9, 103.7, 76.4, 73.6, 71.5, 56.3, 56.2, 53.8, 53.7, 53.4, 32.6, 28.0, 18.4, 18.3, 17.7, 17.5; Found: $[\mathrm{M}+\mathrm{H}]^{+}$, 568.1285. $\mathrm{C}_{24} \mathrm{H}_{30} \mathrm{~N}_{3} \mathrm{O} 8 \mathrm{Br}+\mathrm{H}^{+}$ requires: $[\mathrm{M}+\mathrm{H}]^{+}, 568.1294$.

The quinolone $\mathbf{2 6}$ was isolated as a by-product in variable yield from the reaction of remaining 24 with $\mathrm{TMSCHN}_{2}$. m.p. $179{ }^{\circ} \mathrm{C}$. IR (nujol mull) 3300, $1624 \mathrm{~cm}^{-1} .{ }^{1} \mathrm{H}(300 \mathrm{MHz}$, DMSO) $\delta 9.90(1 \mathrm{H}, \mathrm{br} \mathrm{s}), 7.62(1 \mathrm{H}, \mathrm{d}, J=7.6), 7.53(1 \mathrm{H}, \mathrm{d}, J=7.6), 7.10(1 \mathrm{H}, \mathrm{s}), 7.07(1 \mathrm{H}, \mathrm{t}, J$ = 7.6), $5.96(1 \mathrm{H}, \mathrm{s}), 3.09(1 \mathrm{H}, \mathrm{s}) .{ }^{13} \mathrm{C}(75 \mathrm{MHz}, \mathrm{DMSO}) 161.0,145.0,134.2,131.9,127.4$, 125.5, 124.6, 113.3, 108.0, 75.3, 55.8. HRMS (Found: $\mathrm{M}^{+}, 282.9833 . \mathrm{C}_{11} \mathrm{H}_{10} \mathrm{NO}_{3} \mathrm{Br}^{+}$requires, 282.9844). 


\section{2-Benzyloxycarbonylamino-3-(4-triisopropylsilanyloxy-phenyl)-propionic acid 28.}

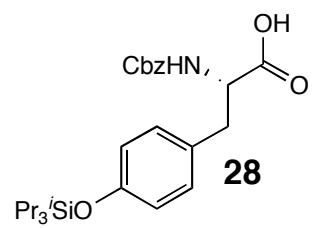

Imidazole (3.72 g, $54.7 \mathrm{mmol})$ and TIPSCl (4.59 mL, $21.7 \mathrm{mmol})$ were added sequentially to a solution of (-)- $N$-Cbz-tyr-methyl ester ${ }^{6}(6.0 \mathrm{~g}, 18.2 \mathrm{mmol})$ in DMF $(70 \mathrm{~mL})$ at $0{ }^{\circ} \mathrm{C}$ under argon. The mixture was stirred at room temperature overnight, concentrated in vacuo, taken up in diethyl ether and water, and extracted three times with diethyl ether. The combined extracts were washed twice with water and with saturated brine, dried $\left(\mathrm{Na}_{2} \mathrm{SO}_{4}\right)$, filtered and concentrated in vacuo to yield TIPS-protected Cbz-Tyr-OMe as a colorless oil. IR 2946, 2867, 1728, $1609 \mathrm{~cm}^{-1}$. ${ }^{1} \mathrm{H}\left(300 \mathrm{MHz}, \mathrm{CDCl}_{3}\right) \delta$ 7.34-7.29 (5H, m), $6.94(2 \mathrm{H}, \mathrm{d}, J=8.5), 6.79(2 \mathrm{H}, \mathrm{d}, J=8.5), 5.21(1 \mathrm{H}$, $\mathrm{d}, J=8.1), 5.10(2 \mathrm{H}, \mathrm{s}), 4.65-4.60(1 \mathrm{H}, \mathrm{m}), 3.69(3 \mathrm{H}, \mathrm{s}), 3.05-3.00(2 \mathrm{H}, \mathrm{m}), 1.31-1.18(3 \mathrm{H}, \mathrm{m})$, $1.10(9 \mathrm{H}, \mathrm{s}), 1.08(9 \mathrm{H}, \mathrm{s}) .{ }^{13} \mathrm{C}\left(75 \mathrm{MHz}, \mathrm{CDCl}_{3}\right) \delta 171.9,155.5,154.9,136.2,130.0,128.3,128.0$, 127.9, 127.9, 119.8, 66.7, 54.9, 52.0, 37.3, 17.7, 12.4. HRMS (Found: $\mathrm{M}^{+}, 485.2599$. $\mathrm{C}_{27} \mathrm{H}_{39} \mathrm{NO}_{5} \mathrm{Si}$ requires 485.2598$) .0 .4 \mathrm{M}$ aq. $\mathrm{KOH}(60 \mathrm{~mL})$ was then added to a solution of the crude TIPS-protected Boc-Tyr-OMe in methanol $(60 \mathrm{~mL})$ and THF $(20 \mathrm{~mL})$ at $0^{\circ} \mathrm{C}$ under argon, and the resulting mixture was allowed to warm slowly to room temperature over 4 hours. The mixture was acidified with $2 \mathrm{M}$ aq. $\mathrm{HCl}$, taken up in ethyl acetate and water, and extracted three times with ethyl acetate. The combined extracts were washed with saturated brine, dried $\left(\mathrm{Na}_{2} \mathrm{SO}_{4}\right)$, filtered and concentrated in vacuo to yield 28 (8.36 g, 97\% overall) which was used in subsequent steps without purification as a colorless oil. $[\alpha]_{\mathrm{D}}{ }^{20}-61.3\left(\mathrm{c}=0.3, \mathrm{CH}_{3} \mathrm{OH}\right) ; R \mathrm{f}(1: 1$ Hexanes:EtOAc) 0.40. IR 3402, 3328, 2944, 1722, $1609 \mathrm{~cm}^{-1} ;{ }^{1} \mathrm{H}\left(300 \mathrm{MHz}, \mathrm{CDCl}_{3}\right)$ 7.26-7.36 $(5 \mathrm{H}, \mathrm{m}), 7.02(2 \mathrm{H}, \mathrm{d}, J=8.4 \mathrm{~Hz}), 6.79(2 \mathrm{H}, \mathrm{d}, J=8.4 \mathrm{~Hz}), 5.21-5.24(1 \mathrm{H}, \mathrm{m}), 5.09(2 \mathrm{H}, \mathrm{s}), 4.75-$ $4.90(2 \mathrm{H}, \mathrm{m}), 4.62-4.67(1 \mathrm{H}, \mathrm{m}) 3.05-3.13(2 \mathrm{H}, \mathrm{m}), 1.26-1.89(3 \mathrm{H}, \mathrm{m}), 1.07(18 \mathrm{H}, \mathrm{d}, \mathrm{J}=7.1 \mathrm{~Hz})$. ${ }^{13} \mathrm{C}\left(75 \mathrm{MHz}, \mathrm{CDCl}_{3}\right) 175.5,156.3,155.6,130.9,128.9,128.6,128.6,128.4,120.5,115.9,68.3$, 67.4, 55.3, 37.4, 18.0, 13.2; m/z (C.I.) 472 (100\%, $\mathrm{M}+\mathrm{H}^{+}$); HRMS (Found: $\mathrm{M}+\mathrm{H}^{+}, 472.2520$. $\mathrm{C}_{26} \mathrm{H}_{38} \mathrm{NO}_{5} \mathrm{Si}$ requires 472.2519). 


\section{5-(7-Bromo-3-hydroxy-1-methoxymethyl-2-oxo-2,3-dihydro-1H-indol-3-yl)-2-\{1-[2-}

benzyloxycarbonylamino-3-(4-triisopropylsilanyloxy-phenyl)-propionylamino]-2methylpropyl\}-oxazole-4-carboxylic acid methyl ester 29.

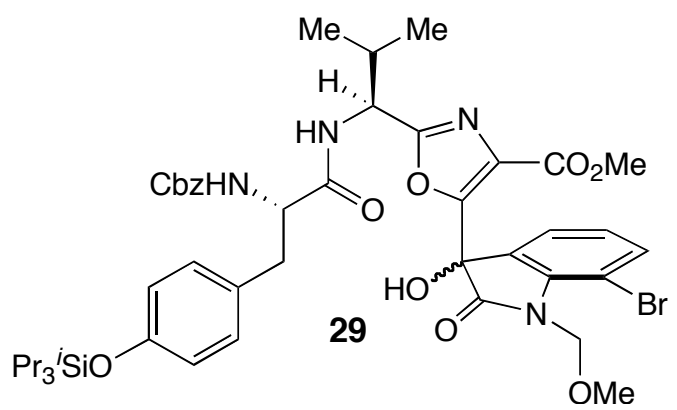

TFA (3 mL) was added to a solution of $\mathbf{2 5}(1.14 \mathrm{~g}, 2 \mathrm{mmol})$ in dichloromethane $(20 \mathrm{~mL})$ at $0{ }^{\circ} \mathrm{C}$ under argon, and the reaction mixture was allowed to warm to room temperature and stirred overnight. The reaction was quenched with saturated aqueous $\mathrm{NaHCO}_{3} / \mathrm{NaHCO}_{3}$, taken up in ethyl acetate and water, and extracted three times with ethyl acetate. The combined extracts were washed with saturated aqueous $\mathrm{NaHCO}_{3}$ and saturated brine, dried $\left(\mathrm{Na}_{2} \mathrm{SO}_{4}\right)$, filtered and concentrated in vacuo to obtain the crude amine intermediate 27. EDC (441 $\mathrm{mg}, 2.3 \mathrm{mmol}$ ) and HOBt. $\mathrm{H}_{2} \mathrm{O}(311 \mathrm{mg}, 2.3 \mathrm{mmol})$ were added to a solution of 28 (1.11 g, $\left.2.35 \mathrm{mmol}\right)$ in DMF (10 $\mathrm{mL}$ ) under argon and the reaction mixture was stirred for 15 minutes. A solution of the crude amine 27 in DMF (5 mL) was added, and the mixture was stirred at room temperature overnight. The solution was partially concentrated in vacuo, taken up in ethyl acetate and water and extracted three times with ethyl acetate. The combined extracts were washed with water and saturated brine, dried $\left(\mathrm{Na}_{2} \mathrm{SO}_{4}\right)$, filtered and concentrated in vacuo. The resulting crude product was purified by flash column chromatography, eluting with 40:60 ethyl acetate:hexane to give 29 (964 mg, 52\% overall) as a mixture of diastereoisomers. m.p. 85-86 ${ }^{\circ}$ C. IR 3297, 2945, 2867, 1748, 1733, 1717, 1699, 1684, 1668, $1662 \mathrm{~cm}^{-1} .{ }^{1} \mathrm{H}\left(300 \mathrm{MHz}, \mathrm{CDCl}_{3}\right) \delta 7.72(0.5 \mathrm{H}, \mathrm{s}), 7.62$ $(0.5 \mathrm{H}, \mathrm{s}), 7.53(0.5 \mathrm{H}, \mathrm{d}, J=7.9), 7.51(0.5 \mathrm{H}, \mathrm{d}, J=8.0), 7.37-7.22(6 \mathrm{H}, \mathrm{m}), 7.03-6.94(3 \mathrm{H}, \mathrm{m})$, $6.76(1 \mathrm{H}, \mathrm{d}, J=8.2), 6.75(1 \mathrm{H}, \mathrm{d}, J=8.2), 6.57-6.44(1 \mathrm{H}, \mathrm{m}), 5.55-5.43(2 \mathrm{H}, \mathrm{m}), 5.22-5.20(1 \mathrm{H}$, m), $5.06(2 \mathrm{H}, \mathrm{s}), 4.91-4.84(1 \mathrm{H}, \mathrm{m}), 4.38-4.30(1 \mathrm{H}, \mathrm{m}), 4.01(1.5 \mathrm{H}, \mathrm{s}), 3.99(1.5 \mathrm{H}, \mathrm{s}), 3.39(1.5 \mathrm{H}$, s), $3.38(1.5 \mathrm{H}, \mathrm{s}), 2.95-2.81(2 \mathrm{H}, \mathrm{m}), 2.07-1.96(1 \mathrm{H}, \mathrm{m}), 1.28-1.14(3 \mathrm{H}, \mathrm{m}), 1.09(9 \mathrm{H}, \mathrm{s}), 1.07$ $(9 \mathrm{H}, \mathrm{s}), 0.76-0.70(6 \mathrm{H}, \mathrm{m}) .{ }^{13} \mathrm{C}\left(75 \mathrm{MHz}, \mathrm{CDCl}_{3}\right) \delta 174.5,174.4,171.1,163.8,163.8,162.2$, 162.2, 155.9, 155.9, 155.2, 155.1, 154.7, 139.6, 139.5, 136.4, 135.8, 130.8, 130.7, 130.0, 129.8, $129.6,128.3,128.2$, 127.8, 127.6, 125.0, 125.0, 124.9, 124.9, 123.9, 119.7, 103.6, 103.5, 73.5, 
73.5, 71.5, 66.7, 66.7, 56.1, 56.0, 55.7, 53.0, 53.0, 52.5, 52.3, 36.9, 36.8, 31.9, 18.3, 18.3, 17.8, 17.7, 17.6, 17.4, 12.3. HRMS (Found: $\mathrm{M}+\mathrm{H}^{+}, 921.3100 . \mathrm{C}_{45} \mathrm{H}_{57} \mathrm{~N}_{4} \mathrm{O}_{10} \mathrm{SiBr}+\mathrm{H}^{+}$requires: , 921.3106).

5-(7-Bromo-3-chloro-1-methoxymethyl-2-oxo-2,3-dihydro-1H-indol-3-yl)-2-\{1-[2benzyloxycarbonylamino-3-(4-triisopropylsilanyloxy-phenyl)-propionylamino]-2methylpropyl\}-oxazole-4-carboxylic acid methyl ester 30 .

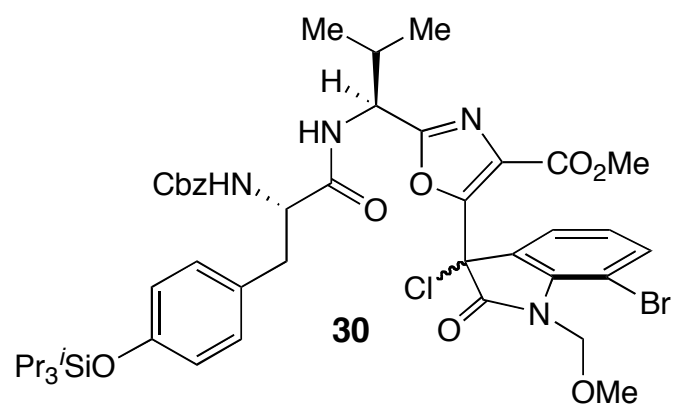

2,4,6-Collidine $(0.829 \mathrm{~mL}, 6.27 \mathrm{mmol})$ and thionyl chloride $(0.228 \mathrm{~mL}, 3.14 \mathrm{mmol})$ were added to a solution of $29(964 \mathrm{mg}, 1.05 \mathrm{mmol})$ in $\mathrm{Et}_{2} \mathrm{O}(10 \mathrm{~mL})$ at $-78^{\circ} \mathrm{C}$ under argon. The mixture was stirred for 10 minutes, then was quenched with water while still cold. The resulting mixture was allowed to warm to room temperature, taken up in ethyl acetate and water and extracted three times with ethyl acetate. The combined extracts were washed with water and with saturated brine, dried $\left(\mathrm{Na}_{2} \mathrm{SO}_{4}\right)$, filtered and concentrated in vacuo. The resulting crude product was purified by flash column chromatography eluting with 30:70 ethyl acetate:hexane to give $\mathbf{3 0}$ (659 mg, 67\%) as a mixture of diastereoisomers. m.p. $69-71{ }^{\circ} \mathrm{C} . R \mathrm{f}(1: 1$ Hexane:EtOAc) 0.8 . IR 3297, 2945, 2867, 1755, 1716, 1662, 1608, $1580 \mathrm{~cm}^{-1} .{ }^{1} \mathrm{H}\left(300 \mathrm{MHz}, \mathrm{CDCl}_{3}\right) \delta 7.53(0.5 \mathrm{H}, \mathrm{d}, J$ $=8.1), 7.53(0.5 \mathrm{H}, \mathrm{d}, J=8.1), 7.36-7.27(5 \mathrm{H}, \mathrm{m}), 7.22(0.5 \mathrm{H}, \mathrm{d}, J=7.5), 7.17(0.5 \mathrm{H}, \mathrm{d}, J=7.4)$, 7.08-7.03 (2H, m), $6.97(0.5 \mathrm{H}, \mathrm{t}, J=7.8), 6.95(0.5 \mathrm{H}, \mathrm{t}, J=7.9), 6.79(1 \mathrm{H}, \mathrm{d}, J=8.3), 6.76(1 \mathrm{H}$, $\mathrm{d}, J=8.3), 6.74-6.68(1 \mathrm{H}, \mathrm{m}), 5.65(1 \mathrm{H}, \mathrm{d}, J=11.0), 5.50(0.5 \mathrm{H}, \mathrm{d}, J=11.0), 5.49(0.5 \mathrm{H}, \mathrm{d}, J=$ 11.0), 5.31-5.24 (1H, m), 5.19-5.14 $(1 \mathrm{H}, \mathrm{m}), 5.08(1 \mathrm{H}, \mathrm{s}), 5.07(1 \mathrm{H}, \mathrm{s}), 4.48-4.45(1 \mathrm{H}, \mathrm{m}), 3.68$ $(1.5 \mathrm{H}, \mathrm{s}), 3.66(1.5 \mathrm{H}, \mathrm{s}), 3.51(3 \mathrm{H}, \mathrm{s}), 3.06(1 \mathrm{H}, \mathrm{d}, J=6.9), 3.04(1 \mathrm{H}, \mathrm{d}, J=7.4), 2.27-2.23(1 \mathrm{H}$, m), 1.29-1.15 (3H, m), $1.09(4.5 \mathrm{H}, \mathrm{s}), 1.08(4.5 \mathrm{H}, \mathrm{s}), 1.06(4.5 \mathrm{H}, \mathrm{s}), 1.05(4.5 \mathrm{H}, \mathrm{s}), 0.94-0.89$ $(6 \mathrm{H}, \mathrm{m}) .{ }^{13} \mathrm{C}\left(75 \mathrm{MHz}, \mathrm{CDCl}_{3}\right) \delta 171.7,171.6,171.0,163.4,163.4,163.0,160.7,160.6,156.0$, 155.1, 155.0, 149.4, 139.5, 136.8, 135.9, 130.5, 130.4, 130.2, 128.4, 128.3, 128.1, 128.0, 127.9, 127.8, 125.2, 123.7, 123.5, 120.1, 120.0, 119.8, 115.5, 103.6, 103.5, 76.6, 72.3, 67.1, 67.0, 59.5, 
56.7, 56.1, 56.0, 55.9, 52.9, 52.8, 52.3, 52.2, 32.6, 32.3, 29.6, 25.8, 18.9, 18.6, 18.1, 17.8, 12.5. HRMS (Found: $\mathrm{M}+\mathrm{H}^{+}, 939.2769 . \mathrm{C}_{45} \mathrm{H}_{56} \mathrm{~N}_{4} \mathrm{O}_{9} \mathrm{SiClBr}+\mathrm{H}^{+}$requires 939.2767).

\section{Cbz-protected macrolactam ethers 31 and 32.}

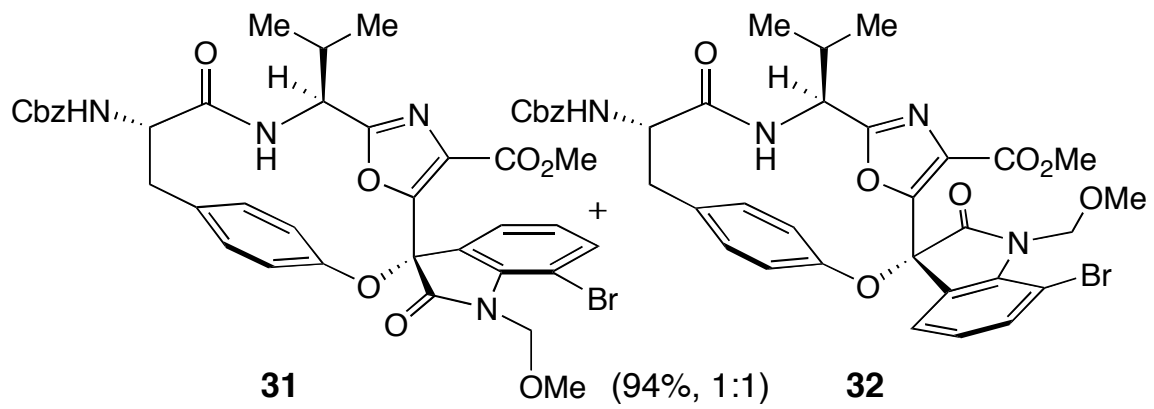

TBAF ( $0.20 \mathrm{~mL}$ of a $1.0 \mathrm{M}$ solution in THF, $0.25 \mathrm{mmol}, 2.3$ equiv.) was added dropwise to a solution of chloride 30 (80 mg, $0.11 \mathrm{mmol}, 1.0$ equiv.) in $\mathrm{THF}(5.0 \mathrm{~mL})$ at $0{ }^{\circ} \mathrm{C}$ under an argon atmosphere. The mixture was stirred at $0{ }^{\circ} \mathrm{C}$ for $10 \mathrm{mins}$ and quenched by the addition of saturated aqueous $\mathrm{NH}_{4} \mathrm{Cl}(1.0 \mathrm{~mL})$. The mixture was extracted with EtOAc $(4 \times 5.0 \mathrm{~mL})$ and the combined organic layers washed with brine $(2 \times 3.0 \mathrm{~mL})$, dried $\left(\mathrm{Na}_{2} \mathrm{SO}_{4}\right)$ and concentrated under reduced pressure. The crude product was purified by flash column chromatography $\left(\mathrm{SiO}_{2} ; 2: 1\right.$ Hexane:EtOAc) to give $\mathbf{3 1}$ and $\mathbf{3 2}$.

$31(30.6 \mathrm{mg}, 48 \%)$ as a white solid: m.p. $192-194{ }^{\circ} \mathrm{C} .[\alpha]_{\mathrm{D}}{ }^{20}+151\left(\mathrm{c}=0.3, \mathrm{CH}_{3} \mathrm{OH}\right)$. $R \mathrm{f}\left(2: 1\right.$ Hexanes:EtOAc) 0.55 . IR 3304, 2955, 1746, 1727, 1712, $1662 \mathrm{~cm}^{-1} .{ }^{1} \mathrm{H}(300 \mathrm{MHz}$, $\left.\mathrm{CDCl}_{3}\right) \delta 7.58(1 \mathrm{H}, \mathrm{d}, J=8.1 \mathrm{~Hz}), 7.44(2 \mathrm{H}, \mathrm{d}, \mathrm{J}=7.4 \mathrm{~Hz}), 7.31-7.36(5 \mathrm{H}, \mathrm{m}), 7.02-7.07(2 \mathrm{H}, \mathrm{t}, J$ $=7.8 \mathrm{~Hz}), 6.95-6.98(2 \mathrm{H}, \mathrm{m}), 5.69(1 \mathrm{H}, \mathrm{d} J=10.8 \mathrm{~Hz}), 5.46(1 \mathrm{H}, \mathrm{d}, J=9.4 \mathrm{~Hz}), 5.40(1 \mathrm{H}, \mathrm{d}, J=$ $8.0 \mathrm{~Hz}), 5.12(2 \mathrm{H}, \mathrm{m}), 4.48(1 \mathrm{H}, \mathrm{t}, J=9.6 \mathrm{~Hz}), 4.10-4.16(1 \mathrm{H}, \mathrm{m}), 3.78(3 \mathrm{H}, \mathrm{s}), 3.48(3 \mathrm{H}, \mathrm{s}), 3.26-$ $3.38(1 \mathrm{H}, \mathrm{m}), 2.62(1 \mathrm{H}, \mathrm{t}, J=11.8 \mathrm{~Hz}), 2.10-2.19(1 \mathrm{H}, \mathrm{m}), 1.22-1.28(1 \mathrm{H}, \mathrm{m}), 0.90(3 \mathrm{H}, \mathrm{d}, J=6.6$ $\mathrm{Hz}), 0.79(3 \mathrm{H}, \mathrm{d}, J=6.6 \mathrm{~Hz}) .{ }^{13} \mathrm{C}\left(75 \mathrm{MHz}, \mathrm{CDCl}_{3}\right)$ 178.4, 173.9, 163.8, 163.4, 156.1, 155.7, $151.7,149.8,140.7,137.2,137.1,134.9,132.5,128.9,128.5,128.2$, 128.0, 124.4, 123.8, 104.1, 102.9, 72.3, 69.3, 67.6, 60.6, 58.3, 56.3, 52.8, 41.0, 31.8, 30.2, 19.8, 19.0, 15.9; m/z (C.I.) 747 (100\%, $\left.\mathrm{M}+\mathrm{H}^{+}\right)$; HRMS (Found: $\mathrm{M}+\mathrm{H}^{+}, 747.1662 . \mathrm{C}_{36} \mathrm{H}_{36} \mathrm{~N}_{4} \mathrm{O}_{9} \mathrm{Br}$ requires 746.1666).

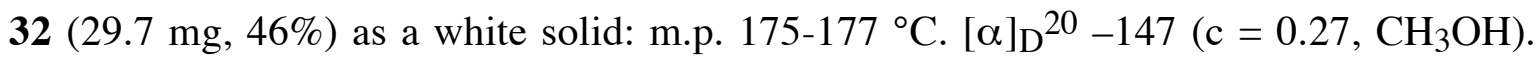
$R f\left(2: 1\right.$ Hexanes:EtOAc) 0.40. IR 3304, 2959, 1747, 1728, 1715, $1662 \mathrm{~cm}^{-1} .{ }^{1} \mathrm{H}(300 \mathrm{MHz}$, $\left.\mathrm{CDCl}_{3}\right) \delta 7.57(1 \mathrm{H}, \mathrm{d}, J=8.0 \mathrm{~Hz}), 7.31-7.42(6 \mathrm{H}, \mathrm{m}), 7.25(1 \mathrm{H}, \mathrm{d}, J=7.6 \mathrm{~Hz}), 7.17(1 \mathrm{H}, \mathrm{dd}, J ' s=$ 
8.4 and $2.7 \mathrm{~Hz}), 7.00(1 \mathrm{H}, \mathrm{t}, J=7.7 \mathrm{~Hz}), 6.82(1 \mathrm{H}, \mathrm{dd}, J ' s=8.6$ and $2.1 \mathrm{~Hz}), 5.74(2 \mathrm{H}, \mathrm{t}, J=7.9$ $\mathrm{Hz}), 5.45(1 \mathrm{H}, \mathrm{d}, J=10.8 \mathrm{~Hz}), 5.17(1 \mathrm{H}, \mathrm{d}, J=12.2 \mathrm{~Hz}), 5.08(1 \mathrm{H}, \mathrm{d}, J=12.2 \mathrm{~Hz}), 4.89-4.92(2 \mathrm{H}$, m), 4.20-4.29 (1H, m), $3.84(3 \mathrm{H}, \mathrm{s}), 3.52(3 \mathrm{H}, \mathrm{s}), 3.37\left(1 \mathrm{H}, \mathrm{dd}, J^{\prime} s=12.2\right.$ and $\left.5.3 \mathrm{~Hz}\right), 2.58(1 \mathrm{H}, \mathrm{t}$, $J=12.2 \mathrm{~Hz}), 1.75-1.84(1 \mathrm{H}, \mathrm{m}), 1.22-1.29(1 \mathrm{H}, \mathrm{m}), 0.89(3 \mathrm{H}, \mathrm{d}, J=6.7 \mathrm{~Hz}), 0.73(3 \mathrm{H}, \mathrm{d}, J=6.7$ Hz). ${ }^{13} \mathrm{C}\left(75 \mathrm{MHz}, \mathrm{CDCl}_{3}\right) \delta 172.3,169.5,161.8,161.2,156.2,155.7,148.9,140.3,137.1,136.1$, $131.8,131.3,128.9,128.5,128.4,128.3,128.2,128.0,125.0,123.9,121.5,119.3,104.1,79.3$, 72.3, 67.0, 60.4, 58.1, 56.7, 52.8, 52.7, 38.8, 32.5, 18.7; $\mathrm{m} / \mathrm{z}$ (C.I.) $747\left(100 \%, \mathrm{M}+\mathrm{H}^{+}\right)$; HRMS (Found: $\mathrm{M}+\mathrm{H}^{+}$, 747.1662. $\mathrm{C}_{36} \mathrm{H}_{36} \mathrm{~N}_{4} \mathrm{O} 9 \mathrm{Br}$ requires 746.1666).

\section{Macrolactam phenols 33 and 34.}
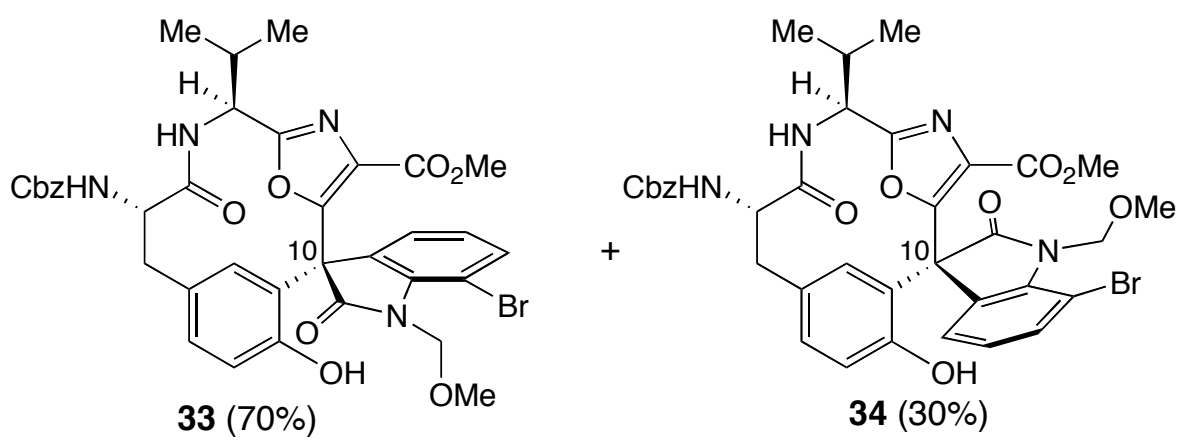

A 1:1 mixture of the diastereomeric ethers $\mathbf{3 1}$ and $\mathbf{3 2}(0.20 \mathrm{~g}, 0.27 \mathrm{mmol}, 1.0$ equiv) were treated to general method $\mathbf{B}$ (solvent $=$ chloroform $(15 \mathrm{~mL})$, temperature $=$ reflux, time $=4 \mathrm{~h}$ ), before being concentrated in vacuo to obtain $\mathbf{3 3}$ and $\mathbf{3 4}(0.14 \mathrm{~g}, 70 \%)$ as a $7: 3$ mixture of diastereoisomers which could be separated by column chromatography $\left(\mathrm{SiO}_{2}:\right.$ 8:2 Dichloromethane:EtOAc).

33; m.p. $170-175{ }^{\circ} \mathrm{C} .[\alpha]_{\mathrm{D}}{ }^{20}-237\left(\mathrm{c}=0.33, \mathrm{CH}_{3} \mathrm{OH}\right) . R \mathrm{f}\left(7: 3 \mathrm{CH}_{2} \mathrm{Cl}_{2}\right.$ :EtOAc) 0.30. IR 3307, 3061, 3028, 2958, 2929, 1727, 1663, 1605, $1575 \mathrm{~cm}^{-1} .{ }^{1} \mathrm{H}\left(300 \mathrm{MHz}, \mathrm{CHCl}_{3}\right) \delta 7.68(1 \mathrm{H}, \mathrm{bs}$, exchanges with $\left.\mathrm{D}_{2} \mathrm{O}\right), 7.48(1 \mathrm{H}, \mathrm{d}, J=8.1), 7.42-7.27(6 \mathrm{H}, \mathrm{m}), 7.06(1 \mathrm{H}, \mathrm{d}, J=7.2), 6.97(1 \mathrm{H}, \mathrm{d}$, $J=6.9), 6.86(1 \mathrm{H}, \mathrm{t}, J=7.8), 6.80-6.78(1 \mathrm{H}, \mathrm{m}), 6.34(1 \mathrm{H}, \mathrm{s}), 5.62(1 \mathrm{H}, \mathrm{d}, J=8.4), 5.51(1 \mathrm{H}, \mathrm{d}, J$ $=10.8), 5.41(1 \mathrm{H}, \mathrm{d}, J=11.1), 5.13(2 \mathrm{H}, \mathrm{s}), 4.81(1 \mathrm{H}, \mathrm{t}, J=6.9), 3.95(1 \mathrm{H}, \mathrm{t}, J=9.0), 3.39(3 \mathrm{H}$, s), $3.34(3 \mathrm{H}, \mathrm{s}), 3.27-3.25(1 \mathrm{H}, \mathrm{m}), 2.72(1 \mathrm{H}, \mathrm{d}, J=11.4), 2.04(1 \mathrm{H}, \mathrm{m}), 1.05(3 \mathrm{H}, \mathrm{d}, J=6.3), 0.86$ $(3 \mathrm{H}, \mathrm{d}, J=6.0) .{ }^{1} \mathrm{H}(300 \mathrm{MHz}, \mathrm{DMSO}) \delta 9.81\left(1 \mathrm{H}, \mathrm{s}\right.$, exchanges with $\left.\mathrm{D}_{2} \mathrm{O}\right), 9.00(1 \mathrm{H}, \mathrm{d}, J=5.7)$, $7.67(1 \mathrm{H}, \mathrm{d}, J=7.2), 7.47(1 \mathrm{H}, \mathrm{d}, J=6.9), 7.35-7.27(6 \mathrm{H}, \mathrm{m}), 6.97(1 \mathrm{H}, \mathrm{d}, J=8.1), 6.89(1 \mathrm{H}, \mathrm{d}, J$ $=6.6), 6.56(1 \mathrm{H}, \mathrm{d}, J=8.1), 6.21(1 \mathrm{H}, \mathrm{s}), 5.42(1 \mathrm{H}, \mathrm{d}, J=11.1), 5.32(1 \mathrm{H}, \mathrm{d}, J=10.8), 5.00(2 \mathrm{H}$, 
d, $J=5.4), 4.35(1 \mathrm{H}, \mathrm{t}, J=7.5), 4.12(1 \mathrm{H}, \mathrm{m}), 3.29(3 \mathrm{H}, \mathrm{s}), 3.15(3 \mathrm{H}, \mathrm{s}), 3.00(1 \mathrm{H}, \mathrm{t}, J=12.0)$, 2.47-2.46 (1H, m, masked by DMSO peak), 1.98-1.96 (1H, m), 0.97 (3H, d, $J=6.3), 0.82(3 \mathrm{H}, \mathrm{d}$, $J=6.3) .{ }^{13} \mathrm{C}\left(75 \mathrm{MHz}, \mathrm{CDCl}_{3}\right) \delta 175.9,171.7,163.3,161.0,156.3,151.6,140.7,135.5,134.9$, $132.3,131.5,130.9,130.8,130.5,128.7,128.5,128.1,126.8,126.0,124.5,123.8,116.0,102.9$, $71.9,67.6,57.2,56.2,55.6,52.5,38.5,30.0,20.1,19.0 .{ }^{13} \mathrm{C}(75 \mathrm{MHz}, \mathrm{DMSO}) \delta 174.2,172.3$, 164.1, 160.9, 155.2, 152.3, 140.3, 137.1, 134.4, 132.1, 131.2, 130.3, 129.8, 128.4, 127.9, 127.8, 127.0, 126.1, 124.5, 123.4, 115.5, 102.0, 71.3, 65.4, 55.9, 55.6, 54.9, 52.0, 29.3, 28.2, 19.7, 19.1. HRMS (Found: $\mathrm{M}+\mathrm{H}^{+}, 747.1660 . \mathrm{C}_{36} \mathrm{H}_{36} \mathrm{~N}_{4} \mathrm{O}_{9} \mathrm{Br}$ requires 747.1666).

34; m.p. $165-168{ }^{\circ} \mathrm{C} .[\alpha]_{\mathrm{D}^{20}}-116\left(\mathrm{c}=0.21, \mathrm{CH}_{3} \mathrm{OH}\right) . R \mathrm{f}\left(7: 3 \mathrm{CH}_{2} \mathrm{Cl}_{2}\right.$ :EtOAc) 0.39. IR 3301, 3060, 3033, 2959, 2929, 1733, 1659, 1608, $1573 \mathrm{~cm}^{-1} .{ }^{1} \mathrm{H}(300 \mathrm{MHz}, \mathrm{DMSO}) \delta 9.43(1 \mathrm{H}, \mathrm{s}$, exchanges with $\left.\mathrm{D}_{2} \mathrm{O}\right), 8.91(1 \mathrm{H}, \mathrm{d}, J=6.3), 7.68(1 \mathrm{H}, \mathrm{d}, J=7.2), 7.46(1 \mathrm{H}, \mathrm{d}, J=7.8), 7.35-7.07$ $(6 \mathrm{H}, \mathrm{m}), 7.06-6.89(2 \mathrm{H}, \mathrm{m}), 6.49(1 \mathrm{H}, \mathrm{d}, J=7.8), 6.01(1 \mathrm{H}, \mathrm{s}), 5.61(1 \mathrm{H}, \mathrm{d}, J=10.8), 5.25(1 \mathrm{H}, \mathrm{d}$, $J=10.8), 5.00(2 \mathrm{H}, \mathrm{s}), 4.43(1 \mathrm{H}, \mathrm{t}, J=6.8), 4.15(1 \mathrm{H}, \mathrm{t}, J=7.8), 3.61(3 \mathrm{H}, \mathrm{s}), 3.25(3 \mathrm{H}, \mathrm{s}), 2.95$ $(1 \mathrm{H}, \mathrm{t}, J=12.0), 2.47-2.46(1 \mathrm{H}, \mathrm{m}$, masked by DMSO peak), 2.06-1.97 $(1 \mathrm{H}, \mathrm{m}), 0.98(3 \mathrm{H}, \mathrm{d}, J=$ 6.0), $0.82(3 \mathrm{H}, \mathrm{d}, J=5.4) .{ }^{13} \mathrm{C}(75 \mathrm{MHz}, \mathrm{DMSO}) \delta 173.4172 .2,163.6,161.0,155.2,153.2$, 139.7, 137.1, 133.8, 133.3, 133.0, 130.2, 130.1, 128.4, 127.8, 127.7, 127.5, 126.4, 124.5, 122.7, 115.6, 114.8, 102.0, 71.3, 65.4, 55.8, 55.5, 55.0, 52.5, 29.2, 28.2, 19.6, 19.0. HRMS (Found: $\mathrm{M}+\mathrm{H}^{+}$, 747.1666. $\mathrm{C}_{36} \mathrm{H}_{36} \mathrm{~N}_{4} \mathrm{O} 9 \mathrm{Br}$ requires 747.1666).

HPLC analysis [Apex ${ }^{\mathrm{TM}}$ silica column (particle size, 5 _ m; length, $150 \mathrm{~mm}$ ); $5 \%$ i-PrOH in hexane for elution; $1 \mathrm{ml} / \mathrm{min}$ flow rate at r.t.; detection at $220 \mathrm{~nm}$ ]; $\mathrm{t}_{\mathrm{R}}=5 \mathrm{~min}, \mathbf{3 1} ; \mathrm{t}_{\mathrm{R}}=7 \mathrm{~min} \mathrm{32}$; $\mathrm{t}_{\mathrm{R}}=18 \min , \mathbf{3 3} ; \mathrm{t}_{\mathrm{R}}=25 \min \mathbf{3 4}$.

\section{Direct Conversion of 30 into 33.}

TBAF (2.30 mL, $1 \mathrm{M}$ in THF, $2.3 \mathrm{mmol}$ ) was added dropwise to a solution of $\mathbf{3 0}$ (930 $\mathrm{mg}, 0.989 \mathrm{mmol})$ in $\mathrm{THF}(30 \mathrm{~mL})$ at $0^{\circ} \mathrm{C}$ under argon. The mixture was stirred for 10 minutes, then was taken up in aq. $\mathrm{NH}_{4} \mathrm{Cl}$ and ethyl acetate, and extracted three times with ethyl acetate. The combined extracts were washed with saturated brine, dried $\left(\mathrm{Na}_{2} \mathrm{SO}_{4}\right)$, filtered and concentrated in vacuo to obtain the crude macrocyclic ethers $\mathbf{3 1}$ and $\mathbf{3 2}$ as a 1:1 mixture of diastereoisomers. The crude mixture of macrocyclic ethers 31/32 were taken up in degassed chloroform $(60 \mathrm{~mL})$ and the solution was heated at reflux for $4 \mathrm{~h}$, concentrated in vacuo and 
purified by flash column chromatography, eluting with 55:45 ethyl acetate:hexane, to obtain the rearranged macrolactam $\mathbf{3 3}$ (518 $\mathrm{mg}, 70 \%$ ) as a white solid.

\section{MOM protected phenol 35 .}

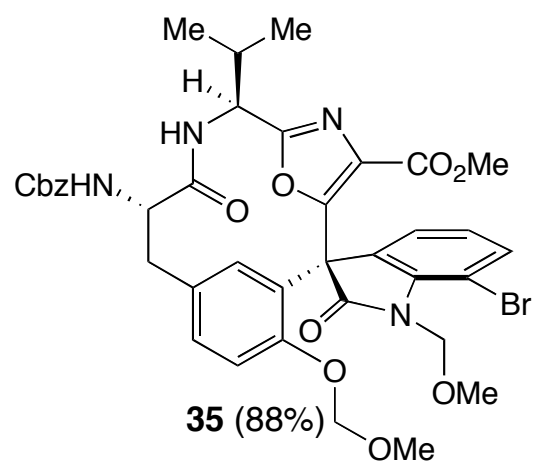

Diisopropylethylamine ( $0.07 \mathrm{~mL}, 0.38 \mathrm{mmol}, 2.0$ equiv.) and $\mathrm{MOMCl}(0.03 \mathrm{~mL}, 0.38$ mmol, 2.0 equiv.) were added dropwise to a solution of $\mathbf{3 4}(0.14 \mathrm{~g}, 0.19 \mathrm{mmol}, 1.0$ equiv.) in $\mathrm{CH}_{2} \mathrm{Cl}_{2}(5 \mathrm{~mL})$ at $0{ }^{\circ} \mathrm{C}$ under an $\operatorname{argon}$ atmosphere. The solution was stirred at $0{ }^{\circ} \mathrm{C}$ for $1.5 \mathrm{~h}$. Water $(3.0 \mathrm{~mL})$ was added and the mixture extracted with EtOAc $(4 \times 5.0 \mathrm{~mL})$. The combined organic layers were washed with brine $(2 \times 3.0 \mathrm{~mL})$, dried $\left(\mathrm{Na}_{2} \mathrm{SO}_{4}\right)$ and concentrated under reduced pressure. The crude product was purified by flash column chromatography $\left(\mathrm{SiO}_{2} ; 1: 1\right.$ Hexane:EtOAc) to give $35(0.13 \mathrm{~g}, 88 \%)$ as a white solid. m.p. $141-143{ }^{\circ} \mathrm{C} .[\alpha]_{\mathrm{D}}{ }^{20}-109(\mathrm{c}=0.45$, $\left.\mathrm{CH}_{3} \mathrm{OH}\right) . R \mathrm{f}\left(1: 1\right.$ Hexanes:EtOAc) 0.45. IR 3315, 2963, 1732, 1708, $1667 \mathrm{~cm}^{-1} .{ }^{1} \mathrm{H}(300 \mathrm{MHz}$, $\left.\mathrm{CDCl}_{3}\right) \delta 7.44\left(1 \mathrm{H}, \mathrm{dd}, J^{\prime} s=8.1\right.$ and $\left.1.2 \mathrm{~Hz}\right), 7.29-7.37(5 \mathrm{H}, \mathrm{m}), 7.14\left(1 \mathrm{H}, \mathrm{dd}, J^{\prime} s=9.8\right.$ and 1.6 $\mathrm{Hz}), 6.96(2 \mathrm{H}, \mathrm{t}, J=8.3 \mathrm{~Hz}), 6.84(1 \mathrm{H}, \mathrm{t}, J=7.8 \mathrm{~Hz}), 6.47-6.50(1 \mathrm{H}, \mathrm{m}), 6.36-6.38(1 \mathrm{H}, \mathrm{m}), 5.58$ $(1 \mathrm{H}, \mathrm{d}, J=10.1 \mathrm{~Hz}), 5.46-5.53(2 \mathrm{H}, \mathrm{m}), 5.12(2 \mathrm{H}, \mathrm{s}), 5.00(1 \mathrm{H}, \mathrm{d}, J=6.8 \mathrm{~Hz}), 4.90(1 \mathrm{H}, \mathrm{d}, J=6.8$ $\mathrm{Hz}), 4.76(1 \mathrm{H}, \mathrm{t}, J=7.4 \mathrm{~Hz}), 4.11-4.18(1 \mathrm{H}, \mathrm{m}), 3.78-3.84(1 \mathrm{H}, \mathrm{m}), 3.46(3 \mathrm{H}, \mathrm{s}), 3.38(3 \mathrm{H}, \mathrm{s}), 3.17$ $(3 \mathrm{H}, \mathrm{s}), 2.72\left(1 \mathrm{H}, \mathrm{dd}, J^{\prime} s=12.7\right.$ and $\left.2.1 \mathrm{~Hz}\right), 2.05-2.16(1 \mathrm{H}, \mathrm{m}), 1.21-1.29(1 \mathrm{H}, \mathrm{m}), 1.06(3 \mathrm{H}, \mathrm{d}, J$ $=6.7 \mathrm{~Hz}), 0.97(3 \mathrm{H}, \mathrm{d}, J=6.7 \mathrm{~Hz}) .{ }^{13} \mathrm{C}\left(75 \mathrm{MHz}, \mathrm{CDCl}_{3}\right)$ 174.6, 172.2, 163.2, 160.8, 155.4, $152.2,151.9,140.5,132.3,131.0,129.9,129.0,128.9,128.6,128.4,128.2,128.0,124.2,123.8$, 114.5, 102.5, 93.9, 71.5, 67.1, 60.4, 57.1, 56.0, 55.9, 55.8, 52.3, 37.9, 30.3, 19.3, 19.1, 14.2. $\mathrm{m} / \mathrm{z}$ (C.I.) $791\left(100 \%, \mathrm{M}^{+}\right)$; HRMS (Found: $\mathrm{M}^{+}$, 791.1916. $\mathrm{C}_{38} \mathrm{H}_{40} \mathrm{~N}_{4} \mathrm{O}_{10} \mathrm{Br}$ requires 791.1928). 
Primary Alcohol 36.

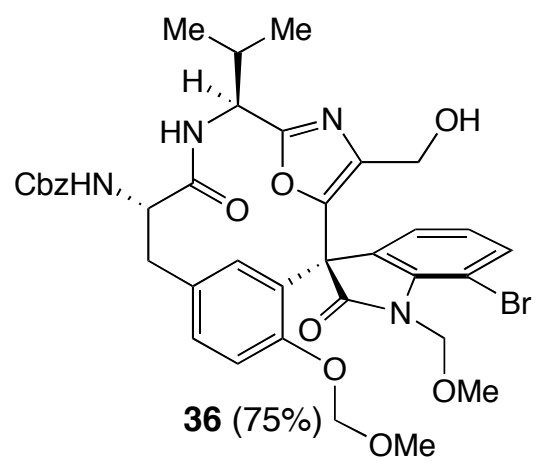

$\mathrm{LiBH}_{4}$ (0.01g. $0.49 \mathrm{mmol}, 3.0$ equiv) was added to a solution of 35 (0.13 g, $0.16 \mathrm{mmol}, 1.0$ equiv) in $\mathrm{THF}(10 \mathrm{~mL})$ at $0{ }^{\circ} \mathrm{C}$ under argon, and the reaction mixture was allowed to warm to room temperature and stirred for 5 hours. The reaction mixture was quenched with sat. aq. $\mathrm{NH}_{4} \mathrm{Cl}$ $(2.5 \mathrm{~mL})$ and a few drops of $2 \mathrm{M} \mathrm{HCl}$, then was taken up in ethyl acetate $(10 \mathrm{~mL})$ and water $(10$ $\mathrm{mL})$ and extracted with ethyl acetate $(3 \times 10 \mathrm{~mL})$. The combined organic layers were washed with sat. brine $(10 \mathrm{~mL})$, dried over sodium sulfate, filtered and concentrated in vacuo. The resulting crude product was purified by flash column chromatography $\left(\mathrm{SiO}_{2}:\right.$ 60:40-100:0 ethyl acetate:hexane) to give 36 (93 mg, 75\%). m.p. $134-136{ }^{\circ} \mathrm{C} .[\alpha]_{\mathrm{D}}{ }^{20}-198\left(\mathrm{c}=0.7, \mathrm{CH}_{3} \mathrm{OH}\right)$. $R f\left(100 \%\right.$ EtOAc) 0.50 . IR 3425, 3308, 2963, 1738, 1708, $1668 \mathrm{~cm}^{-1} .{ }^{1} \mathrm{H}\left(300 \mathrm{MHz}, \mathrm{CDCl}_{3}\right) \delta$ $7.50(1 \mathrm{H}, \mathrm{d}, J=8.0 \mathrm{~Hz}), 7.31-7.38(5 \mathrm{H}, \mathrm{m}), 7.13(1 \mathrm{H}, \mathrm{d}, J=7.1 \mathrm{~Hz}), 7.08\left(1 \mathrm{H}, \mathrm{dd}, J^{\prime} s=8.3\right.$ and $1.4 \mathrm{~Hz}), 6.90-6.97(2 \mathrm{H}, \mathrm{m}), 6.61(1 \mathrm{H}, \mathrm{d}, J=7.1 \mathrm{~Hz}), 6.27(1 \mathrm{H}, \mathrm{d}, J=1.4 \mathrm{~Hz}), 5.81(1 \mathrm{H}, \mathrm{d}, J=8.6$ $\mathrm{Hz}), 5.50(1 \mathrm{H}, \mathrm{d}, J=9.9 \mathrm{~Hz}), 5.44(1 \mathrm{H}, \mathrm{d}, J=9.9 \mathrm{~Hz}), 5.10-5.15(2 \mathrm{H}, \mathrm{m}), 4.95(1 \mathrm{H}, \mathrm{d}, J=6.8$ $\mathrm{Hz}), 4.83(1 \mathrm{H}, \mathrm{d}, J=6.7 \mathrm{~Hz}), 4.67(1 \mathrm{H}, \mathrm{t}, J=8.0 \mathrm{~Hz}), 4.16-4.19(1 \mathrm{H}, \mathrm{m}), 3.78-3.82(1 \mathrm{H}, \mathrm{m}), 3.44$ $(3 \mathrm{H}, \mathrm{s}), 3.20-3.27(2 \mathrm{H}, \mathrm{m}), 3.09(3 \mathrm{H}, \mathrm{s}), 2.78-2.85(1 \mathrm{H}, \mathrm{m}), 2.65-2.71(1 \mathrm{H}, \mathrm{m}), 2.08-2.19(1 \mathrm{H}, \mathrm{m})$, $1.08(3 \mathrm{H}, \mathrm{d}, J=6.6 \mathrm{~Hz}), 0.99(3 \mathrm{H}, \mathrm{d}, J=6.6 \mathrm{~Hz}) .{ }^{13} \mathrm{C}\left(75 \mathrm{MHz}, \mathrm{CDCl}_{3}\right) \delta 175.4,172.4,164.1$, $155.4,152.5,143.0,139.9,138.5,136.1,135.1,132.1,130.0,129.9$, 129.3, 128.9, 128.6, 128.4, 128.2, 128.1, 124.8, 124.4, 114.2, 102.9, 93.7, 71.5, 67.0, 60.4, 56.9, 56.0, 55.8, 54.7, 37.8, 29.9, 19.4, 19.3. $m / z$ (C.I.) $763\left(100 \%, \mathrm{M}+\mathrm{H}^{+}\right)$; HRMS (Found: $\mathrm{M}+\mathrm{H}^{+}, 763.1978 . \mathrm{C}_{37} \mathrm{H}_{40} \mathrm{~N}_{4} \mathrm{O}_{9} \mathrm{Br}$ requires 763.1979$)$. 
Bis-MOM protected OBn macrolactam - Nicolaou compound 37.

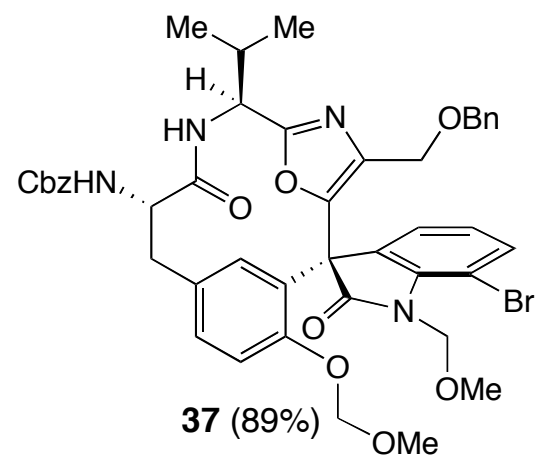

$n$-Bu $4 \mathrm{NI}$ (cat.) and $\mathrm{NaH}(10 \mathrm{mg}, 60 \%$ suspension, $0.16 \mathrm{mmol}, 3.0$ equiv) were added to a solution of 36 (40 mg, $0.052 \mathrm{mmol}, 1.0$ equiv) in 20:80 DMF:THF (1 mL) at $0{ }^{\circ} \mathrm{C}$ under argon and the reaction mixture was stirred for 30 minutes. Benzyl bromide $(0.01 \mathrm{~mL}, 0.052 \mathrm{mmol}, 1.0$ equiv) was added and the reaction mixture was stirred for 45 minutes, still at $0{ }^{\circ} \mathrm{C}$. The reaction was quenched with sat. aq. $\mathrm{NH}_{4} \mathrm{Cl}(0.5 \mathrm{~mL})$, taken up in ethyl acetate $(1.0 \mathrm{~mL})$ and water $(1.0$ $\mathrm{mL})$ and extracted with ethyl acetate $(3 \times 2.0 \mathrm{~mL})$. The combined organic layers were washed with sat. brine $(3.0 \mathrm{~mL})$, dried over sodium sulfate, filtered and concentrated in vacuo. The resulting crude was purified by flash column chromatography $\left(\mathrm{SiO}_{2}: 45: 55\right.$ ethyl acetate:hexane (use 40:60 in future) to obtain $37(40 \mathrm{mg}, 89 \%)$ as an amorphous white solid whose data corresponded with literature data. $[\alpha]_{\mathrm{D}}^{20}-180.7\left(\mathrm{c}=0.7, \mathrm{CH}_{3} \mathrm{OH}\right), \mathrm{Lit}^{5}[\alpha]_{\mathrm{D}}^{20}=-177.0(\mathrm{c}=$ 0.57, $\left.\mathrm{CH}_{3} \mathrm{OH}\right)$. IR 3308, 2960, 2929, 1735, 1717, 1670, $1608 \mathrm{~cm}^{-1} .{ }^{1} \mathrm{H}\left(500 \mathrm{MHz}, \mathrm{CD}_{3} \mathrm{CN}\right) \delta$ $7.52\left(1 \mathrm{H}, \mathrm{dd}, J^{\prime} s=8.2\right.$ and 1.3), 7.36-7.26 (9H, m), 7.21-7.19 (2H, m), $7.14(1 \mathrm{H}, \mathrm{d}, J=7.5), 7.14$ $(1 \mathrm{H}, \mathrm{d}, J=7.5), 6.91(2 \mathrm{H}, \mathrm{t}, J=7.8), 6.35(1 \mathrm{H}, \mathrm{s}), 6.03(1 \mathrm{H}, \mathrm{br} \mathrm{d}, J \sim 5), 5.43(1 \mathrm{H}, \mathrm{d}, J=10.2)$, $5.30(1 \mathrm{H}, \mathrm{d}, J=10.2), 5.08(1 \mathrm{H}, \mathrm{d}, J=12.6), 5.06(1 \mathrm{H}, \mathrm{d}, J=12.6), 4.92(1 \mathrm{H}, \mathrm{d}, J=6.7), 4.88$ $(1 \mathrm{H}, \mathrm{d}, J=6.7), 4.46(1 \mathrm{H}, \mathrm{t}, J=7.7), 4.18-4.03(3 \mathrm{H}, \mathrm{m}), 3.70(1 \mathrm{H}, \mathrm{d}, J=11.6), 3.65(1 \mathrm{H}, \mathrm{d}, J=$ 11.6), $3.30(3 \mathrm{H}, \mathrm{s}), 3.11(1 \mathrm{H}, \mathrm{t}, J=12.5), 3.05(3 \mathrm{H}, \mathrm{s}), 2.66(1 \mathrm{H}, \mathrm{t}, J=11.7), 2.04-2.00(1 \mathrm{H}, \mathrm{m})$, $1.01(3 \mathrm{H}, \mathrm{d}, J=6.2), 0.90(3 \mathrm{H}, \mathrm{d}, J=6.2) .{ }^{13} \mathrm{C}\left(125 \mathrm{MHz}, \mathrm{CD}_{3} \mathrm{CN}\right) 176.2,173.5,164.9,153.0$, $146.0,141.5,139.1,138.2,137.0,136.1,133.6,130.9,130.6,130.5,130.3,129.5,129.3,128.9$, 128.9, 128.7, 128.5, 126.0, 125.1, 114.5, 103.5, 94.4, 73.1, 72.4, 67.1, 64.3, 57.6, 57.2, 56.5, 56.0, 38.3, 30.8, 19.8, 19.4. HRMS (Found: $\mathrm{M}+\mathrm{H}^{+}$, 853.2435. $\mathrm{C}_{44} \mathrm{H}_{45} \mathrm{~N}_{4} \mathrm{O} 9 \mathrm{Br}+\mathrm{H}^{+}$requires 853.2448). 


\section{2-tert-Butoxycarbonylamino-3-(4-triisopropylsilanyloxy-phenyl)-propionic acid $38{ }^{7}$}

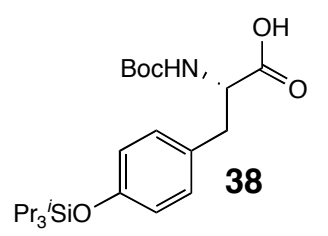

Imidazole (7.85 g, $115.3 \mathrm{mmol})$ and TIPSCl $(9.77 \mathrm{~mL}, 46.1 \mathrm{mmol})$ were added sequentially to a solution of (-)- $N$-Boc-tyr-methyl ester (11.35 g, $38.4 \mathrm{mmol})$ in DMF (150 mL) at $0^{\circ} \mathrm{C}$ under argon. The mixture was stirred at room temperature overnight, concentrated in vасио, taken up in diethyl ether and water, and extracted three times with diethyl ether. The extracts were washed twice with water and with sat. brine, dried $\left(\mathrm{Na}_{2} \mathrm{SO}_{4}\right)$, filtered and concentrated in vacuo to yield TIPS-protected Boc-Tyr-OMe as a colorless oil. IR 2946, 2868, $1747,1717,1609 \mathrm{~cm}^{-1} .{ }^{1} \mathrm{H}\left(300 \mathrm{MHz}, \mathrm{CDCl}_{3}\right) \delta 6.97(2 \mathrm{H}, \mathrm{d}, J=8.4), 6.80(2 \mathrm{H}, \mathrm{d}, J=8.4), 4.95$ $(1 \mathrm{H}, \mathrm{d}, J=7.9), 4.57-4.49(1 \mathrm{H}, \mathrm{m}), 3.68(3 \mathrm{H}, \mathrm{s}), 3.02-2.94(2 \mathrm{H}, \mathrm{m}), 1.42(9 \mathrm{H}, \mathrm{s}), 1.36-1.18(3 \mathrm{H}$, m), $1.10(9 \mathrm{H}, \mathrm{s}), 1.08(9 \mathrm{H}, \mathrm{s}) .{ }^{13} \mathrm{C}\left(75 \mathrm{MHz}, \mathrm{CDCl}_{3}\right) \delta 172.4,155.0,130.1,128.3,119.9,79.7$, 54.5, 52.0, 37.5, 28.2, 17.8, 12.5. HRMS (Found: $\mathrm{M}^{+}, 451.2760 . \mathrm{C}_{24} \mathrm{H}_{41} \mathrm{NO}_{5} \mathrm{Si}^{+}$requires 451.2754).

To a solution of the crude TIPS-protected Boc-Tyr-OMe derivative in methanol $(125 \mathrm{~mL})$ and $\mathrm{THF}(50 \mathrm{~mL})$ at $0^{\circ} \mathrm{C}$ under argon was added $0.4 \mathrm{M}$ aq. $\mathrm{KOH}(125 \mathrm{~mL})$, and the resulting mixture was allowed to warm slowly to room temperature over $4 \mathrm{~h}$. The mixture was acidified with $2 \mathrm{M}$ aq. $\mathrm{HCl}$, taken up in ethyl acetate and water and extracted three times with ethyl acetate. The combined organic layers were washed with saturated brine, dried $\left(\mathrm{Na}_{2} \mathrm{SO}_{4}\right)$, filtered and concentrated in vacuo to yield $\mathbf{3 8}$ (16.73 g, 99\% overall) as a colorless gum which was used in subsequent steps without purification. IR 2945, 2867, 1716, $1610 \mathrm{~cm}^{-1} .{ }^{1} \mathrm{H}\left(300 \mathrm{MHz}, \mathrm{CDCl}_{3}\right) \delta$ $7.03(2 \mathrm{H}, \mathrm{d}, J=8.4), 6.83(2 \mathrm{H}, \mathrm{d}, J=8.4), 4.90-4.87(1 \mathrm{H}, \mathrm{m}), 4.56-4.50(1 \mathrm{H}, \mathrm{m}), 3.16-2.97(2 \mathrm{H}$, m), $1.42(9 \mathrm{H}, \mathrm{s}), 1.37-1.18(3 \mathrm{H}, \mathrm{m}), 1.10-1.05(18 \mathrm{H}, \mathrm{s})$. HRMS (Found: $\mathrm{M}^{+}, 437.2596$. $\mathrm{C}_{23} \mathrm{H}_{39} \mathrm{NO}_{5} \mathrm{Si}$ requires 437.2598). 


\section{5-(7-Bromo-3-hydroxy-1-methoxymethyl-2-oxo-2,3-dihydro-1H-indol-3-yl)-2-\{1-[2-tert-}

butoxycarbonylamino-3-(4-triisopropylsilanyloxy-phenyl)-propionylamino]-2methylpropyl\}-oxazole-4-carboxylic acid methyl ester 39.

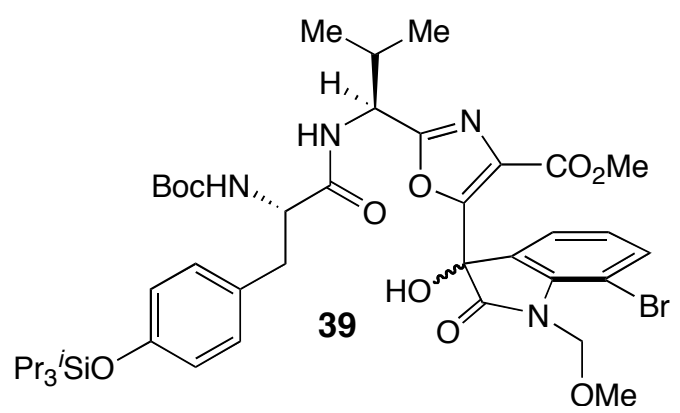

Trifluoroacetic acid (10 mL) was added to a solution 25 (4.67 g, $8.22 \mathrm{mmol})$ in dichloromethane $(60 \mathrm{~mL})$ at $0{ }^{\circ} \mathrm{C}$ under argon, and the mixture was allowed to warm to room temperature and stirred overnight. The reaction was quenched with sat. aq. $\mathrm{NaHCO}_{3}$, taken up in ethyl acetate and water, and extracted three times with ethyl acetate. The combined extracts were washed with sat. aq. $\mathrm{NaHCO}_{3}$ and sat. brine, dried $\left(\mathrm{Na}_{2} \mathrm{SO}_{4}\right)$, filtered and concentrated in vacuo to obtain the crude amine intermediate 27. EDC $(1.73 \mathrm{~g}, 9.04 \mathrm{mmol})$ and $\mathrm{HOBt} . \mathrm{H}_{2} \mathrm{O}(1.22 \mathrm{~g}$, $9.04 \mathrm{mmol})$ were added to a solution of 38 (4.11 g, $9.4 \mathrm{mmol})$ in DMF (40 mL) under argon and the mixture was stirred for 15 minutes. A solution of the crude amine intermediate 27 in DMF (40 mL) was added and the mixture was stirred at room temperature overnight, then was partially concentrated in vacuo, taken up in ethyl acetate and water, and extracted three times with ethyl acetate. The combined organic layers were washed with water and saturated brine, dried $\left(\mathrm{Na}_{2} \mathrm{SO}_{4}\right)$, filtered and concentrated in vacuo. The resulting crude product was purified by flash column chromatography eluting with flash column chromatography $\left(\mathrm{SiO}_{2}: 1: 2\right.$ ethyl acetate:hexane) to give $39(5.36 \mathrm{~g}, 73 \%)$ as a mixture of diastereomers. m.p. $85-87{ }^{\circ} \mathrm{C}$. IR 3308 , 2945, 2868, 1748, 1734, 1716, 1696, 1684, $1609 \mathrm{~cm}^{-1} .{ }^{1} \mathrm{H}\left(300 \mathrm{MHz}, \mathrm{CDCl}_{3}\right) \delta 7.71(0.5 \mathrm{H}, \mathrm{s})$, $7.63(0.5 \mathrm{H}, \mathrm{s}), 7.52(0.5 \mathrm{H}, \mathrm{d}, J=8.1), 7.51(0.5 \mathrm{H}, \mathrm{d}, J=8.0), 7.29(0.5 \mathrm{H}, \mathrm{d}, J=7.4), 7.23(0.5 \mathrm{H}$, $\mathrm{d}, J=7.3), 7.02-6.94(3 \mathrm{H}, \mathrm{m}), 6.77(1 \mathrm{H}, \mathrm{d}, J=8.4), 6.76(1 \mathrm{H}, \mathrm{d}, J=8.4), 6.64(0.5 \mathrm{H}, \mathrm{br} \mathrm{d}, J=$ 8.2), $6.73(0.5 \mathrm{H}$, br d, $J=8.2), 5.54-5.42(2 \mathrm{H}, \mathrm{m}), 4.91-4.85(2 \mathrm{H}, \mathrm{m}), 4.27-4.16(1 \mathrm{H}, \mathrm{m}), 4.01$ $(1.5 \mathrm{H}, \mathrm{s}), 4.00(1.5 \mathrm{H}, \mathrm{s}), 3.38(1.5 \mathrm{H}, \mathrm{s}), 3.37(1.5 \mathrm{H}, \mathrm{s}), 2.93(1 \mathrm{H}, \mathrm{d}, J=6.7), 2.86(1 \mathrm{H}, \mathrm{d}, J=$ 5.9), 2.09-1.98 (1H, m), $1.37(9 \mathrm{H}, \mathrm{s}), 1.27-1.12(3 \mathrm{H}, \mathrm{m}), 1.08(9 \mathrm{H}, \mathrm{s}), 1.05(9 \mathrm{H}, \mathrm{s}), 0.79-0.70$ $(6 \mathrm{H}, \mathrm{m}) .{ }^{13} \mathrm{C}\left(75 \mathrm{MHz}, \mathrm{CDCl}_{3}\right) \delta 174.6,174.4,171.1,171.1,164.2,164.1,162.3,162.2,155.4$, 
155.3, 155.2, 154.8, 139.6, 139.5, 136.5, 130.8, 130.6, 130.1, 130.0, 129.9, 128.5, 125.1, 125.0, 124.1, 124.0, 119.9, 103.7, 103.7, 80.1, 77.1, 73.6, 73.6, 71.6, 71.5, 56.2, 56.1, 55.4, 53.3, 53.2, 52.4, 52.2, 36.4, 36.3, 36.3, 32.3, 28.1, 28.0, 18.3, 18.2, 17.8, 17.7, 12.8, 12.4. HRMS (Found: $\mathrm{M}+\mathrm{H}^{+}$, 887.3293. $\mathrm{C}_{42} \mathrm{H}_{60} \mathrm{~N}_{4} \mathrm{O}_{10} \mathrm{SiBr}+\mathrm{H}^{+}$requires 887.3262).

\section{5-(7-Bromo-3-chloro-1-methoxymethyl-2-oxo-2,3-dihydro-1H-indol-3-yl)-2-\{1-[2-tert- butoxycarbonylamino-3-(4-triisopropylsilanyloxy-phenyl)-propionylamino]-2- methylpropyl\}-oxazole-4-carboxylic acid methyl ester 40.}

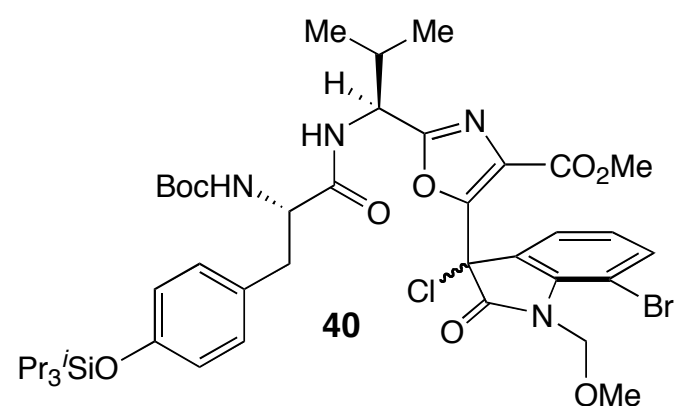

2,4,6-Collidine (7.11 mL, $53.8 \mathrm{mmol})$ and thionyl chloride $(1.96 \mathrm{~mL}, 26.9 \mathrm{mmol})$ were added to a solution of $39(7.96 \mathrm{~g}, 8.96 \mathrm{mmol})$ in $\mathrm{Et}_{2} \mathrm{O}(90 \mathrm{~mL})$ at $-78{ }^{\circ} \mathrm{C}$ under argon. The reaction mixture was stirred for 10 minutes, and quenched with water $(10 \mathrm{~mL})$ while still cold. The resulting mixture was allowed to warm to room temperature, taken up in ethyl acetate $(20 \mathrm{~mL})$ and water $(20 \mathrm{~mL})$ and extracted with ethyl acetate $(3 \times 20 \mathrm{~mL})$. The combined extracts were washed with water $(20 \mathrm{~mL})$ and with sat. brine $(20 \mathrm{~mL})$, dried $\left(\mathrm{Na}_{2} \mathrm{SO}_{4}\right)$, filtered and concentrated in vacuo. The resulting crude product was purified by flash column chromatography $\left(\mathrm{SiO}_{2}: 30: 70\right.$ ethyl acetate:hexane) to obtain give $\mathbf{4 0}(5.69 \mathrm{~g}, 70 \%)$ as a mixture of diastereoisomers. m.p. $82-86^{\circ} \mathrm{C}$. IR $3305,2945,2867,1756,1717,1699,1683,1661,1608$, $1580 \mathrm{~cm}^{-1} .{ }^{1} \mathrm{H}\left(300 \mathrm{MHz}, \mathrm{CDCl}_{3}\right) \delta 7.52(1 \mathrm{H}, \mathrm{d}, J=8.1), 7.22(0.5 \mathrm{H}, \mathrm{d}, J=7.3), 7.16(0.5 \mathrm{H}, \mathrm{d}$, $J=7.3), 7.08(1 \mathrm{H}, \mathrm{d}, J=8.2), 7.06(1 \mathrm{H}, \mathrm{d}, J=8.1), 6.96(0.5 \mathrm{H}, \mathrm{t}, J=7.9), 6.95(0.5 \mathrm{H}, \mathrm{t}, J=7.8)$, $6.81(1 \mathrm{H}, \mathrm{d}, J=8.3), 6.78(1 \mathrm{H}, \mathrm{d}, J=8.2), 6.80-6.70(1 \mathrm{H}, \mathrm{m}), 5.65(1 \mathrm{H}, \mathrm{d}, J=11.0), 5.49(0.5 \mathrm{H}$, d, $J=11.0), 5.48(0.5 \mathrm{H}, \mathrm{d}, J=11.0), 5.20-5.14(1 \mathrm{H}, \mathrm{m}), 4.95(0.5 \mathrm{H}, \mathrm{br} \mathrm{d}, J=5.7), 4.90(0.5 \mathrm{H}, \mathrm{br}$ d, $J=6.7), 4.37-4.32(1 \mathrm{H}, \mathrm{m}), 3.68(1.5 \mathrm{H}, \mathrm{s}), 3.67(1.5 \mathrm{H}, \mathrm{s}), 3.50(3 \mathrm{H}, \mathrm{s}), 3.06-3.00(2 \mathrm{H}, \mathrm{m})$, 2.31-2.22 (1H, m), $1.40(4.5 \mathrm{H}, \mathrm{s}), 1.38(4.5 \mathrm{H}, \mathrm{s}), 1.30-1.14(3 \mathrm{H}, \mathrm{m}), 1.08-1.06(18 \mathrm{H}, \mathrm{m}), 1.00-$ $0.91(6 \mathrm{H}, \mathrm{m})$. HRMS (Found: $\mathrm{M}+\mathrm{H}^{+}, 905.2960 . \mathrm{C}_{42} \mathrm{H}_{58} \mathrm{~N}_{4} \mathrm{O}_{9} \mathrm{SiClBr}+\mathrm{H}^{+}$requires 905.2923). 


\section{Boc-protected macrocyclic etherlactams 41 and 42.}
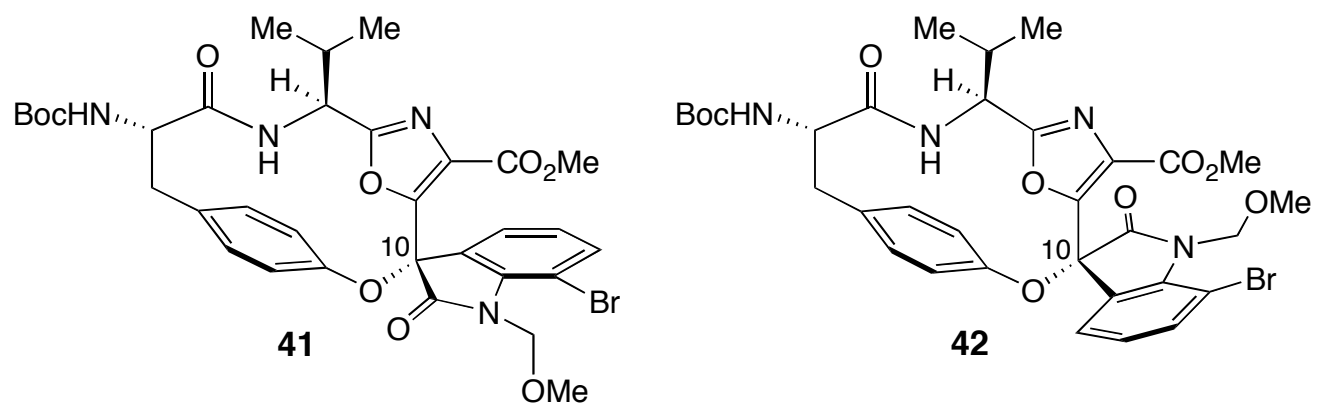

TBAF (7.50 mL, $1 \mathrm{M}$ in THF, $7.50 \mathrm{mmol})$ was added dropwise to a solution of 40 (3.40 $\mathrm{g}, 3.75 \mathrm{mmol})$ in $\mathrm{THF}(65 \mathrm{~mL})$ at $0{ }^{\circ} \mathrm{C}$ under argon. The mixture was stirred for 10 minutes, taken up in aq. $\mathrm{NH}_{4} \mathrm{Cl}(10 \mathrm{~mL})$ and ethyl acetate $(20 \mathrm{~mL})$, and extracted with ethyl acetate $(3 \mathrm{x}$ $20 \mathrm{~mL})$. The combined extracts were washed with saturated brine $(25 \mathrm{~mL})$, dried $\left(\mathrm{Na}_{2} \mathrm{SO}_{4}\right)$, filtered and concentrated in vacuo to give $\mathbf{3 0}$ and $\mathbf{3 1}$ as a 1:1 mixture of diastereoisomers. For spectroscopic analysis a sample was purified by flash column chromatography $\left(\mathrm{SiO}_{2}: 36: 64\right.$ ethyl acetate:hexane) to give $\mathbf{4 1}$ and $\mathbf{4 2}$ as white solids.

41; m.p. 85-105 ${ }^{\circ} \mathrm{C}$, followed by decomposition at $180{ }^{\circ} \mathrm{C} .{ }^{1} \mathrm{H}\left(300 \mathrm{MHz}, \mathrm{CDCl}_{3}\right) \delta 7.58$ $(1 \mathrm{H}, \mathrm{d}, J=7.9), 7.44-7.42(2 \mathrm{H}, \mathrm{m}), 7.06-6.99(4 \mathrm{H}, \mathrm{m}), 5.69(1 \mathrm{H}, \mathrm{d}, J=10.9), 5.43(1 \mathrm{H}, \mathrm{d}, J=$ 10.9), 5.31 (1H, d, $J=9.5), 5.23$ (1H, d, $J=8.4), 4.47(1 \mathrm{H}, \mathrm{t}, J=9.7), 4.08-4.00(1 \mathrm{H}, \mathrm{m}), 3.78$ (3H, s), $3.48(3 \mathrm{H}, \mathrm{s}), 3.25(1 \mathrm{H}, \mathrm{dd}, J=12.2,6.5), 2.59(1 \mathrm{H}, \mathrm{t}, J=12.2), 2.19-2.11(1 \mathrm{H}, \mathrm{m}), 1.44$ $(9 \mathrm{H}, \mathrm{s}), 0.91(3 \mathrm{H}, \mathrm{d}, J=6.7), 0.80(3 \mathrm{H}, \mathrm{d}, J=6.6)$. Found: $\mathrm{M}+\mathrm{H}^{+}, 713.1825$. $\mathrm{C}_{33} \mathrm{H}_{37} \mathrm{~N}_{4} \mathrm{O} 9 \mathrm{Br}+\mathrm{H}^{+}$requires: $\mathrm{M}+\mathrm{H}^{+}, 713.1822$.

42; m.p. $110-125^{\circ} \mathrm{C}$ (began to melt) at $140{ }^{\circ} \mathrm{C}$ resolidified and then melted at $220{ }^{\circ} \mathrm{C}$, $250{ }^{\circ} \mathrm{C}(\mathrm{dec}) .{ }^{1} \mathrm{H}\left(300 \mathrm{MHz}, \mathrm{CDCl}_{3}\right) \delta 7.58(1 \mathrm{H}, \mathrm{d}, J=7.9), 7.32(1 \mathrm{H}, \mathrm{d}, J=8.4), 7.31(1 \mathrm{H}, \mathrm{d}, J$ $=8.5), 7.25(1 \mathrm{H}, \mathrm{d}, J=7.9), 7.15(1 \mathrm{H}, \mathrm{dd}, J=8.4,2.3), 7.01(1 \mathrm{H}, \mathrm{t}, J=7.9), 6.81(1 \mathrm{H}, \mathrm{dd}, J=$ $8.5,1.8), 5.76(1 \mathrm{H}, \mathrm{d}, J=11.0), 5.46-5.40(2 \mathrm{H}, \mathrm{m}), 4.94(1 \mathrm{H}, \mathrm{dd}, J=9.3,7.5), 4.80(1 \mathrm{H}, \mathrm{d}, J=$ 9.9), 4.22-4.13 (1H, m), $3.85(3 \mathrm{H}, \mathrm{s}), 3.52(3 \mathrm{H}, \mathrm{s}), 3.34(1 \mathrm{H}, \mathrm{dd}, J=12.3,5.5), 2.55(1 \mathrm{H}, \mathrm{t}, J=$ 12.3), 1.87-1.76 (1H, m), $1.46(9 \mathrm{H}, \mathrm{s}), 0.88(3 \mathrm{H}, \mathrm{d}, J=6.7), 0.74(3 \mathrm{H}, \mathrm{d}, J=6.7)$. HRMS (Found: $\mathrm{M}+\mathrm{H}^{+}$, 713.1833. $\mathrm{C}_{33} \mathrm{H}_{37} \mathrm{~N}_{4} \mathrm{O}_{9} \mathrm{Br}+\mathrm{H}^{+}$requires 713.1822). 


\section{Boc-protected macrolactams 43 and 44.}
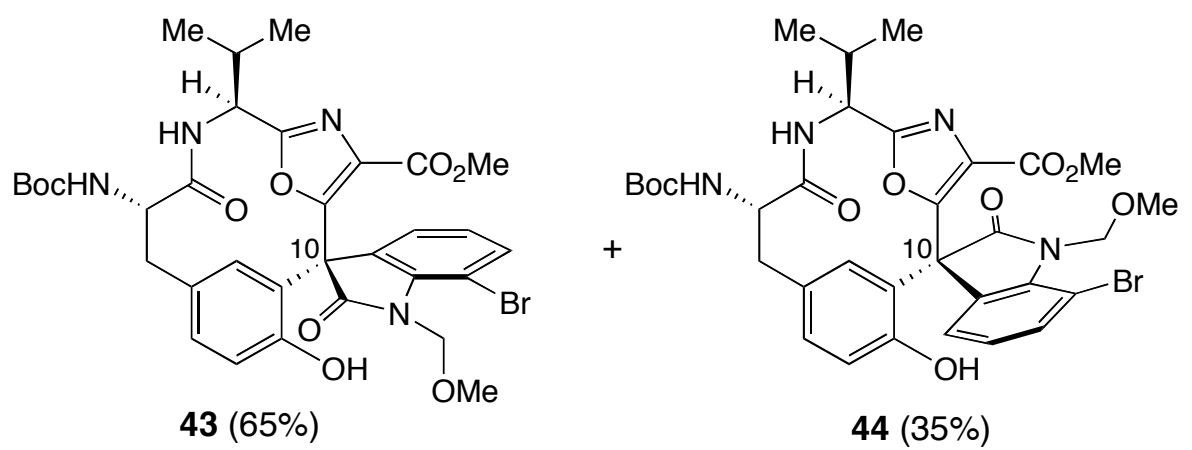

The crude mixture of the macrocyclic ethers 41 and 42 were treated to general method $B$ $($ solvent $=$ chloroform $(200 \mathrm{~mL})$, temperature $=$ reflux, time $=6 \mathrm{~h})$, before being concentrated in vacuo to obtain 43 and 44 (2.22 g, 83\% overall) as a 7:3 mixture of diastereoisomers which could be separated by column chromatography $\left(\mathrm{SiO}_{2}: 8: 2\right.$ Dichloromethane:EtOAc).

43. m.p. $>195^{\circ} \mathrm{C}(\mathrm{dec}) \cdot[\alpha]_{\mathrm{D}}{ }^{20}-242\left(\mathrm{c}=0.7, \mathrm{CH}_{3} \mathrm{OH}\right) . R \mathrm{f}\left(7: 3 \mathrm{CH}_{2} \mathrm{Cl}_{2}\right.$ :EtOAc) 0.28. IR 3320, 2967, 2928, 1737, 1728, 1670, 1608, 1577, $1510 \mathrm{~cm}^{-1} .{ }^{1} \mathrm{H}\left(300 \mathrm{MHz}, \mathrm{CDCl}_{3}\right) \delta 8.36(1 \mathrm{H}$, br s, exchanges with $\left.\mathrm{D}_{2} \mathrm{O}\right), 7.77\left(1 \mathrm{H}, \mathrm{d}, J=6.6\right.$, moves to 8.20 in $\left.\mathrm{CDCl}_{3} / \mathrm{D}_{2} \mathrm{O}\right), 7.44(1 \mathrm{H}, \mathrm{d}, J=7.8)$, $7.08(1 \mathrm{H}, \mathrm{d}, J=7.2), 6.95(1 \mathrm{H}, \mathrm{d}, J=7.2), 6.87-6.82(2 \mathrm{H}, \mathrm{m}), 6.32(1 \mathrm{H}, \mathrm{s}), 5.50-5.46(3 \mathrm{H}, \mathrm{m})$, $4.76(1 \mathrm{H}, \mathrm{t}, J=7.2), 3.94(1 \mathrm{H}, \mathrm{t}, J=9.3), 3.38(3 \mathrm{H}, \mathrm{s}), 3.34(3 \mathrm{H}, \mathrm{s}), 3.17(1 \mathrm{H}, \mathrm{t}, J=12.0), 2.66$ (1H, br d, $J=11.1), 2.02-1.98(1 \mathrm{H}, \mathrm{m}), 1.44(9 \mathrm{H}, \mathrm{s}), 1.03(3 \mathrm{H}, \mathrm{d}, J=6.6), 0.85(3 \mathrm{H}, \mathrm{d}, J=6.6)$. ${ }^{1} \mathrm{H}(300 \mathrm{MHz}, \mathrm{DMSO}) \delta 9.78\left(1 \mathrm{H}, \mathrm{s}\right.$, exchanges with $\left.\mathrm{D}_{2} \mathrm{O}\right), 8.93-8.91(1 \mathrm{H}, \mathrm{m}), 7.47(1 \mathrm{H}, \mathrm{dd}, J=$ 6.9, 2.1), 7.03-6.85 (4H, m), $6.56(1 \mathrm{H}, \mathrm{d}, J=8.1), 6.21(1 \mathrm{H}, \mathrm{s}), 5.42(1 \mathrm{H}, \mathrm{d}, J=10.5), 5.32(1 \mathrm{H}$, $\mathrm{d}, J=10.5), 4.36(1 \mathrm{H}, \mathrm{t}, J=6.6), 4.05(1 \mathrm{H}, \mathrm{bt}, J=8.4), 3.29(3 \mathrm{H}, \mathrm{s}), 3.15(3 \mathrm{H}, \mathrm{s}), 2.95(1 \mathrm{H}, \mathrm{t}, J$ = 12.0), 2.47-2.44 (1H, m, masked by DMSO peak), 1.98-1.96 (1H, m), $1.36(9 \mathrm{H}, \mathrm{s}), 0.97(3 \mathrm{H}$, $\mathrm{d}, J=6.0), 0.82(3 \mathrm{H}, \mathrm{d}, J=6.6) .{ }^{13} \mathrm{C}\left(75 \mathrm{MHz}, \mathrm{CDCl}_{3}\right) \delta 176.0,172.2,163.5,160.9,155.7$, $151.9,151.4,140.6,134.7,132.2,131.2,130.9,130.5,127.0,126.0,124.4,123.6,116.2$, 102.8, 81.1, 71.9, 56.6, 56.2, 55.5, 52.3, 38.6, 30.0, 28.2, 19.8, 18.9. ${ }^{13} \mathrm{C}$ (75 MHz, DMSO) $\delta ~ 174.2$, $172.5,164.1$, 160.8, 154.5, 152.2, 151.8, 140.3, 134.4, 132.0, 131.3, 130.2, 129.7, 127.1, 126.0, $124.5,123.3,115.4,101.9,78.0,71.3,55.9,55.6,54.8,51.9,38.7,29.2,28.2$, 19.7, 19.0. HRMS (Found: $\mathrm{M}+\mathrm{H}^{+}, 713.1819 . \mathrm{C}_{33} \mathrm{H}_{37} \mathrm{~N}_{4} \mathrm{O}_{9} \mathrm{Br}+\mathrm{H}^{+}$requires 713.1822).

44. m.p. $>220{ }^{\circ} \mathrm{C}$ (dec). $[\alpha]_{\mathrm{D}}{ }^{20}-110\left(\mathrm{c}=0.7, \mathrm{CH}_{3} \mathrm{OH}\right) . R f\left(7: 3 \mathrm{CH}_{2} \mathrm{Cl}_{2}\right.$ :EtOAc) 0.34. IR 3320, 2967, 2928, 2870, 1723, 1666, 1607, 1572, $1507 \mathrm{~cm}^{-1} .{ }^{1} \mathrm{H}\left(300 \mathrm{MHz}, \mathrm{CDCl}_{3}\right) \delta 7.44(1 \mathrm{H}, \mathrm{d}, J=$ 
8.1), 7.01-6.93 (2H, bm), $6.85(2 \mathrm{H}, \mathrm{t}, J=7.8), 6.69-6.54(2 \mathrm{H}, \mathrm{bm}), 6.43(1 \mathrm{H}, \mathrm{bs}), 5.73-5.31(3 \mathrm{H}$, bm), 4.81-4.78 (1H, bm), 4.13-4.08 (1H, bs), $3.71(3 \mathrm{H}, \mathrm{s}), 3.35(3 \mathrm{H}, \mathrm{bs}), 3.11-3.01(1 \mathrm{H}, \mathrm{bm})$, 2.63-2.57 (1H, bm), 2.18-2.10 (1H, bm), $1.46(9 \mathrm{H}, \mathrm{s}), 1.05-0.99(6 \mathrm{H}, \mathrm{bm}) .{ }^{1} \mathrm{H}(300 \mathrm{MHz}$, DMSO) $\delta 9.42\left(1 \mathrm{H}, \mathrm{s}\right.$, exchanges with $\left.\mathrm{D}_{2} \mathrm{O}\right), 8.83(1 \mathrm{H}, \mathrm{d}, J=5.7), 7.45(1 \mathrm{H}, \mathrm{d}, J=7.8), 7.02-$ $7.00(2 \mathrm{H}, \mathrm{m}), 6.94(2 \mathrm{H}, \mathrm{d}, J=7.5), 6.49(1 \mathrm{H}, \mathrm{d}, J=7.8), 6.03(1 \mathrm{H}, \mathrm{s}), 5.60(1 \mathrm{H}, \mathrm{d}, J=10.8)$, $5.26(1 \mathrm{H}, \mathrm{d}, J=10.5), 4.43(1 \mathrm{H}, \mathrm{t}, J=7.2), 4.12-4.06(1 \mathrm{H}, \mathrm{bm}), 3.59(3 \mathrm{H}, \mathrm{s}), 3.25(3 \mathrm{H}, \mathrm{s}), 2.92-$ $2.86(1 \mathrm{H}, \mathrm{m}), 2.47(1 \mathrm{H}, \mathrm{m}$, masked by DMSO $), 2.07-2.02(1 \mathrm{H} \mathrm{m}), 1.37(9 \mathrm{H}, \mathrm{s}), 0.98(3 \mathrm{H}, \mathrm{d}, J=$ 5.4), $0.84(3 \mathrm{H}, \mathrm{d}, J=6.3) .{ }^{13} \mathrm{C}(75 \mathrm{MHz}, \mathrm{DMSO}) \delta 173.4,172.3,163.6,161.0,154.6,153.1$, 152.9, 139.7, 133.8, 133.2, 133.0, 130.0, 128.5, 126.6, 124.5, 124.3, 122.8, 115.6, 102.0, 78.3, 71.3, 55.5, 55.3, 55.0, 51.8, 37.0, 29.1, 28.2, 19.5, 19.0. HRMS (Found: $\mathrm{M}+\mathrm{H}^{+}, 713.1821$. $\mathrm{C}_{33} \mathrm{H}_{37} \mathrm{~N}_{4} \mathrm{O}_{9} \mathrm{Br}+\mathrm{H}^{+}$requires 713.1822).

HPLC analysis [Apex ${ }^{\mathrm{TM}}$ silica column (particle size, 5 _m; length, $150 \mathrm{~mm}$ ); $5 \%$ i-PrOH in hexane for elution; $1 \mathrm{ml} / \mathrm{min}$ flow rate at r.t.; detection at $220 \mathrm{~nm}] ; \mathrm{t}_{\mathrm{R}}=4 \mathrm{~min}, \mathbf{4 1} ; \mathrm{t}_{\mathrm{R}}=6 \mathrm{~min} \mathrm{42}$; $\mathrm{t}_{\mathrm{R}}=12 \min , \mathbf{4 3} ; \mathrm{t}_{\mathrm{R}}=19 \min \mathbf{4 4}$.

\section{References and Footnotes}

$\dagger \quad$ Author for inquiries concerning the X-ray data.

(1) Meyers, A. I., Tavares, F. X. J. Org. Chem. 1996, 61, 8207-8215.

(2) Burgess, E.M.; Penton, H.R.; Taylor, E.A.; Williams, W. M. Org. Syntheses Coll. Vol VI, 1988, 788-790.

(3) Williams, D. R.; Lowder, P. D.; Gu, Y.-G.; Brooks, D. A. Tetrahedron Lett. 1997, 38, 331-334. Downing, S.V.; Aguilar, E.; Meyers, A.I. J. Org. Chem. 1999, 64, 826-831.

(4) Newman, M.S.; Logue, M.W. J. Org. Chem. 1971, 36, 1398-1401. Modified using chloral alcoholate. Acros 34653 CAS [515-83-3].

(5) Nicolaou, K. C.; Chen, D. Y.-K.; Huang, X.; Ling, T.; Bella, M.; Snyder, S. A. J. Am. Chem. Soc. 2004, 126, 12888-12896.

(6) Lin, S.; Yang, Z-Q.; Kwok, B. H. B.; Koldobskiy, M.; Crews, C. M.; Danishefsky, S. J. J. Am. Chem Soc. 2004, 126, 6347-6355.

(7) Wasserman, H. H.; Zhang, R. Tetrahedron 2002, 58, 6277-6284. 